\title{
Exploring Trading Strategies and their Effects in the FX Market
}

\author{
Monira Aloud $^{1}$, Maria Fasli ${ }^{2}$ \\ March 16, 2016 \\ ${ }^{1}$ College of Business Administration, King Saud University, KSA \\ ${ }^{2}$ School of Computer Science and Electronic Engineering, University of Essex, UK \\ Email: mealoud@ksu.edu.sa,mfasli@essex.ac.uk
}




\begin{abstract}
One of the most critical issues that developers face in developing automatic systems or software agents for electronic markers is that of endowing the agents with appropriate trading strategies. In this paper, we examine the problem in the Foreign Exchange (FX) market and we use an agent-based FX market simulation to examine which trading strategies lead to market states in which the stylized facts (statistical properties) of the simulation match the stylised facts of the actual FX market transactions data. In particular, our goal is to explore the emergence of the stylized facts of the transactions data, when the simulated market is populated with agents using three different strategies: a variation of the zero-intelligence with a constraint (ZI-CV) strategy; the zero-intelligence directional-change event (ZI-DCT0) strategy; and a genetic programmingbased (GP) strategy. A series of experiments were conducted in an existing agent-based FX market with these three strategies and the results were compared against those of a high-frequency transactions dataset from the FX market. Our results show that the ZI-DCT0 agents best reproduce and explain the properties and phenomena observed in the FX market real transactions data. Our study suggests that the observed stylized facts of the FX market transactions data could be the result of introducing a threshold which triggers the agents to respond to fixed periodic patterns in the price time series. The results of this study can be used further to develop decision support systems and autonomous trading agent strategies for the FX market.
\end{abstract}

Keywords: agent-based modelling, agent-based simulation, electronic markets, FX markets, trading strategies. 


\section{Introduction}

To study and analyse financial and other markets, researchers attempt to identify the statistical properties of the market data. The identified statistical properties of the financial market data are referred to as stylized facts Dacorogna et al. (2001). The difference between classical economics theories and the empirical stylized facts identified from the financial markets data are the main motivating force for the advance of diverse approaches to studying the behaviour of such markets. For example, the concept of bounded rationality has completely replaced the concept of full rational homogeneous representative agents Simon (1982, 1995); Arthur (1991). Such a change in conception inspires the need to study and revise some of the existing economic theories which are based on many idealised assumptions.

Agent-based markets (ABMs) have emerged as an alternative approach for exploring and studying dynamic behaviour in financial and other markets, especially where the application of analytical models is difficult and their use may not be able to yield satisfactory results or useful insights LeBaron (2006); Martinez-Jaramillo and Tsang (2009). An introduction to ABMs is presented in LeBaron (2001) while overviews of the most influential works in the field of ABMs are provided in LeBaron (2006); Samanidou et al. (2007).

ABMs that emulate the behaviour of real markets need to be modelled in such a way so as to exhibit the same stylized facts as real ones LeBaron (2006). In an ABM, the observation of the same stylized facts as the real market serves as benchmark and evidence that the ABM is indeed closely modelling the real market with a high degree of confidence. ABMs exhibiting the same stylized facts as real markets can be used to identify and interpret their origins through comprehensive experimentation; this can provide useful insights into the workings of the real market, the forces that drive behaviour and their impact on the market dynamics which would not otherwise be possible LeBaron (2001). Developing an in depth understanding of the market workings through stylized facts is fundamental for developing more effective decision support systems. A number of ABMs have been able to exhibit some of the markets' stylized facts Alfi et al. (2009); MartinezJaramillo and Tsang (2009); Arthur et al. (1997); Daniel (2006), whereas only some of these ABMs have clearly defined and interpreted the origins of the market stylized facts, such as the works in Daniel (2006); Alfi et al. (2009); Martinez-Jaramillo and Tsang (2009).

Although ABMs are a powerful tool for modelling markets and understanding their behaviour, they have often been criticised for their underlying complexity Hommes (2006). Simplicity is considered essential LeBaron (2006); Hommes (2006); Samanidou et al. (2007), as a complex design may impede the study, understanding and interpretation of the stylized facts' origins Hommes (2006). Though it is often tempting to develop ABMs with intricate set ups and numerous composite parameters, such, often unwarranted complexity, makes it difficult to identify which elements are accountable for the emergence of stylized facts and whether all of the elements are equally important in reproducing them LeBaron (2006). One of the key design questions is the representation and structure of the agents' strategies LeBaron (2001). The analysis and understanding of real traders' behaviour and expectations is one of the most difficult tasks in understanding market behaviour Dacorogna et al. (2001). The representation of strategies may range from simple budget constrained zerointelligence (ZI) agents Daniel (2006); Duffy and Unver (2006); Gode and Sunder (1993a), to complicated intelligent agents Martinez-Jaramillo and Tsang (2009); Cliff and Bruten (1997a). Different strategies suppose different means of identifying arbitrage opportunities LeBaron (2001). The fundamental question becomes what is the simplest strategy that would lead to the emergence of the same stylized facts in the ABM as the real market. An interesting related question is whether learning is necessary and important in such strategies. In designing decision support systems for e-markets, this is also a key question. The paper seeks to understand this, and investigates the impact of three strategy designs on the emergence of the Foreign Exchange (FX) market transactions stylized facts in an agent-based FX market. To this end, we aim to shed light to the problem of designing trading strategies for the FX market.

This paper extends the work in Aloud et al. (2012) where we have constructed an ABM of the FX market (ABFXM) which is able to reproduce, to a certain extent, the stylized facts of the FX market transactions data, and using a systematic approach, we examined the implications of varying the market's features and the impact on reproducing the stylized facts. In this paper, we examine the impact of different strategies on the emergence of the stylized facts in the ABFXM and their respective performance in such a market given a real dataset of historical prices. Such a study can inform the design of agent trading strategies and decision support systems for the FX market. In particular, we examine trading agents with three different strategies: a variation of the ZI with a budget constraint (ZI-CV) strategy, the ZI directional-change event (ZI-DCT0) strategy and a genetic 
programming-based (GP) strategy. The ZI-CV agents trade randomly, subject to budget constraints. The ZIDCT0 agents detect periodic patterns of a fixed size in the price time series. The GP-based agents (GPAs) use genetic programming to forecast price changes and adapt to events in the market. The choice of strategies is motivated (a) by the desire to explore the tradeoff between the use of randomness and artificial intelligence techniques in modelling the agents and (b) by the choice of strategies in previous related work. Strategies similar to the ZI-CV strategy have been used in past studies Daniel (2006); Rayner et al. (2011); Tseng et al. (2010); Duffy and Unver (2006) to explain the emergence of stylized facts in financial markets. The ZI-DCT0 is inspired and based on the work of Alfi et al. (2009), but addresses the limitations of that strategy. The GP-based strategy has parallel characteristics to those of ZIP agents and was chosen as such strategies have been used in previous related work in the FX markets Martinez-Jaramillo and Tsang (2009); Serguieva et al. (2011).

The organization of the rest of the paper is as follows. Section 2 presents a discussion of related work. In section 3, we describe the ABM of the FX market introduced in Aloud et al. (2012). Section 4 provides a description of the three types of trading agent strategies. In section 5, we report on the experiments undertaken in the ABFXM that we developed and the three strategies. The paper closes with a discussion and the conclusions, and avenues for future work.

\section{Related Work}

One of the aims of developing agent-based models of financial markets is to identify and understand the origins of the observed stylized facts of real market behaviour Samanidou et al. (2007). Intrinsically, and as the market consists of multiple interacting agents, one would expect that the design of the agents' trading strategies would have a significant impact on the emergence of the stylized facts LeBaron (2001). The inextricable link between trading strategies and market characteristics has been studied in diverse settings. For instance, Niu et al. (2012, 2010); Robinson et al. (2012) have used the framework of the TAC CAT Market Design Game to study the relation between market dynamics and the agents' adaptive strategies. From a developer/designer point of view it is essential that the agents' trading strategies are clear and simple in order to facilitate analytical tractability, and the interpretation and understanding of the impact of individual behaviour on the market LeBaron (2006); Hommes (2006).

The design of agent strategies for electronic markets and decision support has been the focus of attention of many works for a variety of market settings LeBaron (2001); Alvim and MilidiÃ ${ }^{o}$ (2013); Sanglier et al. (1994); Kettera et al. (2009); Ren et al. (2009). From a modelling perspective, Sim Sim (2002) described a market driven model for designing agents (buyers or sellers) that applies to a wide range of bargaining situations where agents need to reach a consensus. This model takes into account several factors including time, opportunity, competition and eagerness. More recently, this model has been extended for multiple resources negotiation $A n$ et al. (2011).

One of the earliest, leading and most influential ABMs was the Santa Fe Artificial Stock Market (SF ASM) Arthur et al. (1997). The SF ASM can be viewed as a population of intelligent agents, wherein such agents learn and forecast future price movements using a classifier forecasting system. Although, the SF ASM exhibits some of the stock market's stylized facts LeBaron (2006), due to the large number and complexity of the parameters used in modelling the agents' trading strategies, it is not possible to identify and interpret their origin Ladley (2010). A number of works have expanded and modified the SF ASM in terms of the agents' trading strategies such as in Martinez-Jaramillo and Tsang (2009). However, as yet, the origins of the stylized facts remain opaque and indefinable Ladley (2010).

A number of ABMs have made use of what they are known as Zero-Intelligence (ZI) agents to interpret various phenomena in markets Becker (1962); Daniel (2006); Gode and Sunder (1993a). Before the advent of agent-based computational economics, Becker (1962) first introduced participants that behave randomly and irrationally in markets. He demonstrated that some market features such as the slopes of the demand and supply function were the results of the market mechanism which ruled the interaction of the participants' random behavior.

The ZI agent was introduced by Gode and Sunder (1993a) to examine the continuous double-auction (CDA) mechanism. A ZI agent trades randomly, subject to budget constraints. Therefore, ZI agents have no intelligence in the sense that they do not observe nor learn the trend movements in terms of price. The aim of Gode and Sunder's work was to examine the CDA mechanism and to demonstrate clearly the effect of the agents' 
trading strategy and rationality. From their experimental results, they found that markets operating with human traders and ZI constrained agents converged to the equilibrium price while the market operating with ZI unconstrained agents did not. Furthermore, the comparative results showed that markets operating with ZI constrained agents behaved similarly to the market operating with human traders. In subsequent work, Gode and Sunder (1993b) examined the lower-bounds of the level of intelligence or learning agents required to achieve adequate outcomes. They were able to show that a simple budget constrained ZI strategy is able to achieve highly satisfactory outcomes, and that intelligence in the form of learning is not necessary. Their results show that the theoretical equilibrium price in financial markets is determined more by market structure than by the level of intelligence of the traders. The study of Daniel (2006) shows that the stylized facts of the intraday price changes exhibited by real financial markets can be reproduced with ZI agents.

Cliff and Bruten (1997a) challenged the hypothesis of Gode and Sunder (1993b,a) showing different failures in the ability of ZI agents to achieve desirable outcomes. They argue that an approach more intelligent than ZI agents is required to reproduce a real trader's behaviour in a CDA. Cliff and Bruten (1997b) subsequently introduced the Zero-Intelligence Plus (ZIP) strategy which they show reproduces more accurately the behaviour of real traders. ZIP agents can be viewed as a modified version of ZI in which the agents use heuristic rules and simple machine learning techniques to forecast investment opportunities to make a decision for operating in the market.

Tseng et al. (2010) conducted a series of experiments on a web-based market platform to study the properties of real financial markets. In their work, three strategies were built: the ZI, ZIP and the Gjerstad-Dickhaut (GD) auction strategy. The GD strategy is a memory-based agent architecture in which agents place orders based on maximising the expected profit. This relies on the GD agents forming a belief and payoff function. Tseng et al. found that although the ZI strategy is the most naive, ZI agents are the best among the ZIP and GD strategies in terms of describing the stylized facts observed in real financial markets. Their study emphasised the importance of a continuously evolving dynamic trading strategy which implies features similar to that of the ZI model.

Rayner et al. (2011) showed that when agents imitate each other in the market (social learning), this results in the exhibition of the long memory phenomenon. The results show that a non-learning contrarian model of agents who perform a dynamic switching trading strategy produces a more accurate result in terms of long memory than models which imply learning in the agents' trading strategy. Therefore, they conclude that modelling agents' learning is neither sufficient nor necessary for the reproduction of the long memory phenomenon. The work byRen et al. (2009) proposed a model for designing an agent's strategy that makes adaptable rates of concession by negotiating based on the changes in the environment. The proposed model is able to generate sensible decisions to inform agents' strategies in a dynamic environment.

Concerns have been raised regarding the use of the ZI approach and computational intelligence techniques to model the agents' trading strategies Cliff and Bruten (1997a); Ladley (2010); Tseng et al. (2010); Cliff and Bruten (1997b). The randomness in the ZI strategy prevents us from identifying and understanding the reasons behind the dynamics of trading activities. On the other hand, the use of computational intelligence techniques creates a source of complexity and makes analytical tractability of the agents' behaviour more difficult.

Alfi et al. in Alfi et al. (2009) model the agents' strategy using a simple learning mechanism which depends on observing the price's trend movements. Specifically, each agent commits to a fixed threshold, and therefore the agent places an order when the price fluctuations are above this threshold. Due to the simplicity and explicitness of the learning mechanism, the authors defined and interpreted the origins of the stylized facts associated with price returns. They suggested that introducing a threshold which triggers the agent's activities based on observing the price movements, is one of the factors responsible for the emergence of stylized facts. However, the strategy by Alfi et al. does not consider the direction and the overshoot of the price trend where the overshoot represents the magnitude of the price movement beyond the threshold used by the agent.

In this paper, we focus on studying the impact of trading strategies designs in the emergence of such stylized facts in the ABFXM with the view of using the results to inform the development of appropriate trading strategies for the FX market. 


\section{Overview of the Agent-Based FX Market}

In this section, we provide an overview of the agent-based FX market (ABFXM) Aloud et al. (2012) which was developed to simulate the trading activity at the level of an FX market-maker high-frequency market. For a more detailed description we refer the reader to Aloud et al. (2012).

The FX market is where the exchange of currencies, by means of buying and selling, occurs. It is considered the largest, most liquid and most active financial market in the world and is not an individual market but composed of a global network of FX markets Dacorogna et al. (2001). Moreover, it is a decentralized market as there is no central physical marketplace. It operates 24 hours a day, 7 days a week and participants can be governments, central and commercial banks, institutional investors, individual investors, etc. Most FX trading firms are market-makers Dacorogna et al. (2001). A market-maker is a corporation which supplies liquidity for a number of currency pairs, and consequently quotes both a buy and a sell price for a currency pair on its platform. The market-maker buys from and sells to its investors and other market-makers and hence makes a profit on the bid-offer spread.

The ABFXM developed consists of agents that participate in the market by means of buying and selling currencies. For simplicity, there is one currency pair (EUR/USD) available for trading. Each agent will be able to hold, at time $t$, two different types of asset: a risk free asset (cash), and a risky asset (currency). The agents in the ABFXM are modelled with three different strategies as explained in the following section. In addition to the strategy, and in order to provide for a realistic representation of the financial market traders, we have chosen to model heterogeneity and hence the agents: (a) are endowed with different amounts of wealth; (b) possess diverse expectations and preferences, (c) possess different levels of risk appetite, (d) use different trading time windows and (e) place orders of varying size in the market.

Wealth represents the amount of cash that an agent holds and it is expressed in the home currency of the agent's account. Many ABMs assume that all agents are endowed with the same amount of cash prior to the launch of the simulation, as has been done by Daniel (2006); Martinez-Jaramillo and Tsang (2009). However, such an assumption is unrealistic. Over a century ago, Pareto found through empirical observation, that the income distribution in numerous countries follows a power-law distribution Pierre (1965). We quantify the income distribution in financial markets by the income distribution of a country's population. Hence, we use a power law distribution to endow each agent with a different amount of cash which is a more accurate reflection of what happens in reality. A power law distribution requires a minimum $\left(10^{3}\right)$ and a maximum $\left(10^{9}\right)$ boundary for the amount of cash, along with a scaling exponent equal to 1.2 which characterizes the agents' initial wealth distribution.

Every agent $a$ has a portfolio which is expressed in its home currency and records the results of its transactions. The portfolio records the amount of cash and unrealised profits and losses that would be produced if an open position were closed at the current currency pair exchange rate. The Net Asset Value (NAV) of agent $a$ at time $t$ denoted by $N A V_{a, t}$ represents the current value of an account which is the amount of cash in the account plus all unrealized profits and minus all unrealized losses associated with all the account's open positions.

The margin which is in use at time $t$ is denoted by $m_{a, t}$ and it is equal to the position value multiplied by the agent's margin ratio, summed up over all the agent's open positions. The margin available for a new trade is equal to the agent's $N A V_{a, t}$ minus the margin used $m_{a, t}$. Trading on margins enables a trader to place an order when its size is larger than its cash holding; this involves borrowing a fraction of the cash required from the market-maker executing the transaction and depends on the trader's leverage ratio. A leverage ratio of $v: 1$ requires a minimum margin $m \%\left(\frac{1}{v}\right)$ : if a trader decides to open a new position, it must have at least $m \%$ of the position's size available as a margin. Trading on the margin may possibly realize high profits, but this in turn involves high risk. Margin trading makes use of a course of action so-called a margin call. Equation 1 illustrates how to compute the margin call for an agent $a$ 's open position. ${ }^{1}$

$$
N A V_{a, t} \leq \frac{m_{a, t}}{2}
$$

Although the FX market operates on a 24 hours basis, the FX market centres' business hours play an important role in the level of activity in the market. Hence, in our simulation, each agent is associated with local market business trading hours corresponding to its geographical location. These are Sydney, Tokyo,

\footnotetext{
${ }^{1}$ The margin call formula used by OANDA Ltd. trading platform (whose data this study uses) requires a minimum margin of $2 \%$ (or maximum leverage of 50:1).
} 
London and New York. This means that although an agent can trade at any time during the day, the event of doing so outside its business hours is restricted based on a very small probability equal to $10^{-6}$ (except for executing limit orders).

The order size is an important element in our simulation since it is associated with the cautiousness of the agents. In the ABM literature, there are two main approaches to determining a single order size placed by an agent. The direct approach entails an agent's order size being proportional to its wealth Samanidou et al. (2007). This approach creates an extremely high trading risk, given that an agent invests its total cash in a single

order. The second approach assumes that an agent's order size is a fraction of its cash Martinez-Jaramillo and Tsang (2009). The fraction value is determined by a constant trading proportion parameter. In our ABFXM, the determination of the number of currency units of an order depends on the agent's available margin. If Units Available $(U A)$ is the maximum number of currency units an agent $a$ is allowed to trade based on its available margin, then the number of units that will be placed for an order is determined randomly within the specified range $[U A \times 10 \%, U A]$. Equation 2 clarifies how to calculate agent $a$ 's currency units available (UA) to trade.

$$
U A_{a, t}=\frac{M A_{a, t}}{v_{a} \times r}
$$

where $M A_{a, t}$ is the margin available for agent $a ; v_{a}$ is the leverage used by agent $a ; r$ is the exchange rate of the base currency of the currency pair.

There are two types of order, namely, market and limit orders. A market order is an order for direct execution in the market at the present price of the asset. A limit order is an order in which an agent specifies the price at which it is willing to trade. There are two types of limit order: profit taking and stop loss limit orders. The former is an order for closing an open position to lock an assured profit realization. The latter is an order for closing an open position once a particular loss threshold is reached. The reason for a stop loss limit order is to limit the losses of an investor.

The clearance mechanism of the ABFXM involves a very straightforward task. All the market orders at time $t$ will be entirely executed, while limit orders will be executed when their constraints are satisfied. Afterwards, an update of the agent's portfolio will occur for each agent that has an executable order at time $t$. After that, the market's time turns from $t$ to $t+1$. Accordingly, the bid and ask prices are adjusted to the prices at time $t+1$ using the currency pair dataset of the historical bid and ask prices. Based on the recent bid and ask prices, the portfolio will be updated for each agent that holds an open position at time $t+1$. Eventually each open position at time $t+l$ will be checked for a margin call, which is a procedure to close out the open position held by an agent as soon as the amount of cash in its account cascades under the minimum margin needed to cover the size of its currently open position. The intention of the margin call is to prevent an agent from losing more than the amount of cash available in its account.

We restrict the quantity of positions held by an agent $a$ at time $t$ to be one opened position and we assume that a position cannot be adjusted.The market does not imply fees for the transactions. Such simplification assumptions aim to reduce the complexity of the behaviour of the ABFXM to a level that can be studied within the scope of this work. Simplicity is essential to plainly identify the agents' trading behaviour. Therefore, allocating variable quantities results in a significant complication of the market analysis. The relaxation of these three assumptions does not affect the generality of the simulation results presented in our work. Nevertheless, we are aware of the consequence of the role of quantity as a choice variable, mainly with risk aversion.

\section{Agent Strategies}

This section describes the three agent strategies that have been implemented in our ABFXM: a variation of the ZI with a constraint (ZI-CV) strategy, the ZI directional-change event (ZI-DCT0) strategy and the genetic programming-based (GP) strategy. The ZI-CV agents place random orders, subject to budget constraints while the ZI-DCT0 agents detect periodic patterns of a fixed size in the price time series. In contrats, the GP-based agents (GPAs) use genetic programming to forecast price changes in the market.

\subsection{ZI-CV Agents}

Zero-Intelligence with a Constraint (ZI-C) agents as introduced by Gode and Sunder Gode and Sunder (1993a) were designed for a continuous double auction (CDA) market. Although ZI-C agents trade randomly, they do so 
subject to budget constraints to avoid losses. During the market run, for a given unit, the bid and offer prices are drawn from a uniform distribution between the limit price of the unit and either a minimum allowable bid or a maximum allowable offer price Gode and Sunder (1993a). In this work, we use a variation of the ZI-C approach and we call it ZI-CV since the characteristics of the FX market are different to those of the CDA. ZI-CV agents trade randomly with a probability $\lambda \in[0,1]$ which is drawn independently from a uniform distribution, subject to budget constraints in order to avoid investment losses. Therefore, a ZI-CV agent decides to either place a buy or a sell order, or to hold with equal probability ${ }^{2}$. ZI-CV agents are able to place profit-taking and stop-loss limit orders in turn to lock in a certain profit realization or seek to limit the loss potential for an investment.

\subsection{ZI-DCT0 Agents}

The zero-intelligence directional-change event trading strategy (ZI-DCT0), as described in Aloud et al. (2011), is constructed from the directional-change event approach which is used for studying financial time series. The core concept of ZI-DCT0 is that agents will observe the changes in the price time series based on intrinsic time rather than on physical time. Physical time adopts a point based system in which the basic time unit for observing the price changes would range from seconds to hours or days, and therefore time is homogeneous Dacorogna et al. (2001). Studying the price time series using physical time makes tracking changes of the price movements discontinuous, which runs the risk of missing many investment opportunities Dacorogna et al. (2001). In contrast, intrinsic time adopts an event based system where an event is the basic unit Glattfelder et al. (2011). An event is characterized by a threshold, and is defined as the magnitude of a price movement exceeding the threshold Glattfelder et al. (2011). Intrinsic time is irregularly-spaced since time triggers only at periodic events, independent of the notion of physical time Glattfelder et al. (2011). Thus, intrinsic time captures periodic patterns and eliminates irrelevant details of the price change. Most importantly, intrinsic time reduces the complexity of analysing the price time series, and hence reduces the computational cost (i.e. the cost of analysing and studying the price time series) which must not be ignored Glattfelder et al. (2011). For a more in depth analysis of the limitations of physical time and the significance of intrinsic time for studying financial time series we refer interested readers to Aloud et al. (2011).

Given a threshold $\Delta x$, the absolute price change between two local minimum and maximum prices is decomposed into a directional-change (DC) event of size $\Delta x$ and its accompanying overshoot (OS) event. A DC event is usually followed by a price overshoot event rather than a DC event in the reverse direction Glattfelder et al. (2011). The OS event represents the price movement beyond the directional-change event of size $\Delta x$ Glattfelder et al. (2011). A DC event can be one of the two forms: an upturn or downturn DC event Tsang (2010). An upward run is a period between an upturn DC event and the next downturn DC event, whereas a downward run is a period between a downturn DC event and the next upturn DC event Tsang (2010). Prior to the study of an asset's price time series, two variables are defined: the last high and low prices which are set to the asset's price at the start of the sequence. For the period of an upward (downward) run, the last high (low) of asset $s$ is continuously updated to the maximum (minimum) of (a) the current price of asset $s$ and (b) the last high (low) price. An upturn (downturn) DC event concludes a downward (upward) run, and commences an upward (downward) run Tsang (2010). Given a threshold $\Delta x$, a downturn (upturn) DC event occurs once the absolute price change between the current price of asset $s$ and the last high (low) price is lower (higher) than $\Delta x$. A given price threshold of size $\Delta x$ discretizes the price time series into a sequence of

\section{$\cdots \rightarrow$ downturn DC event $\rightarrow$ \\ downturn OSevent $\rightarrow$ \\ upturn DC event $\rightarrow$ \\ upturn OSevent $\rightarrow$ \\ downturn DC event $\rightarrow \cdots$}

Before the launch of the market, based on a continuous uniform distribution, each ZI-DCT0 agent assigns a fixed threshold. Two different equal groups of agents are identified based on ZI-DCT0: contrary agents and trend-following agents. A ZI-DCT0 agent who engages in contrarian behaviour opens a new position on the forecast that the price trend will move in the contrary direction to the current trend. For example, a ZI-DCT0 contrarian agent would open a new long position (buy order) when a downturn DC event of size $\Delta x$ occurs, and after that would close the position (sell order) as soon as the price of the asset rises by a predefined percentage.

\footnotetext{
${ }^{2}$ This is a $33 \%$ equal split among the three actions.
} 


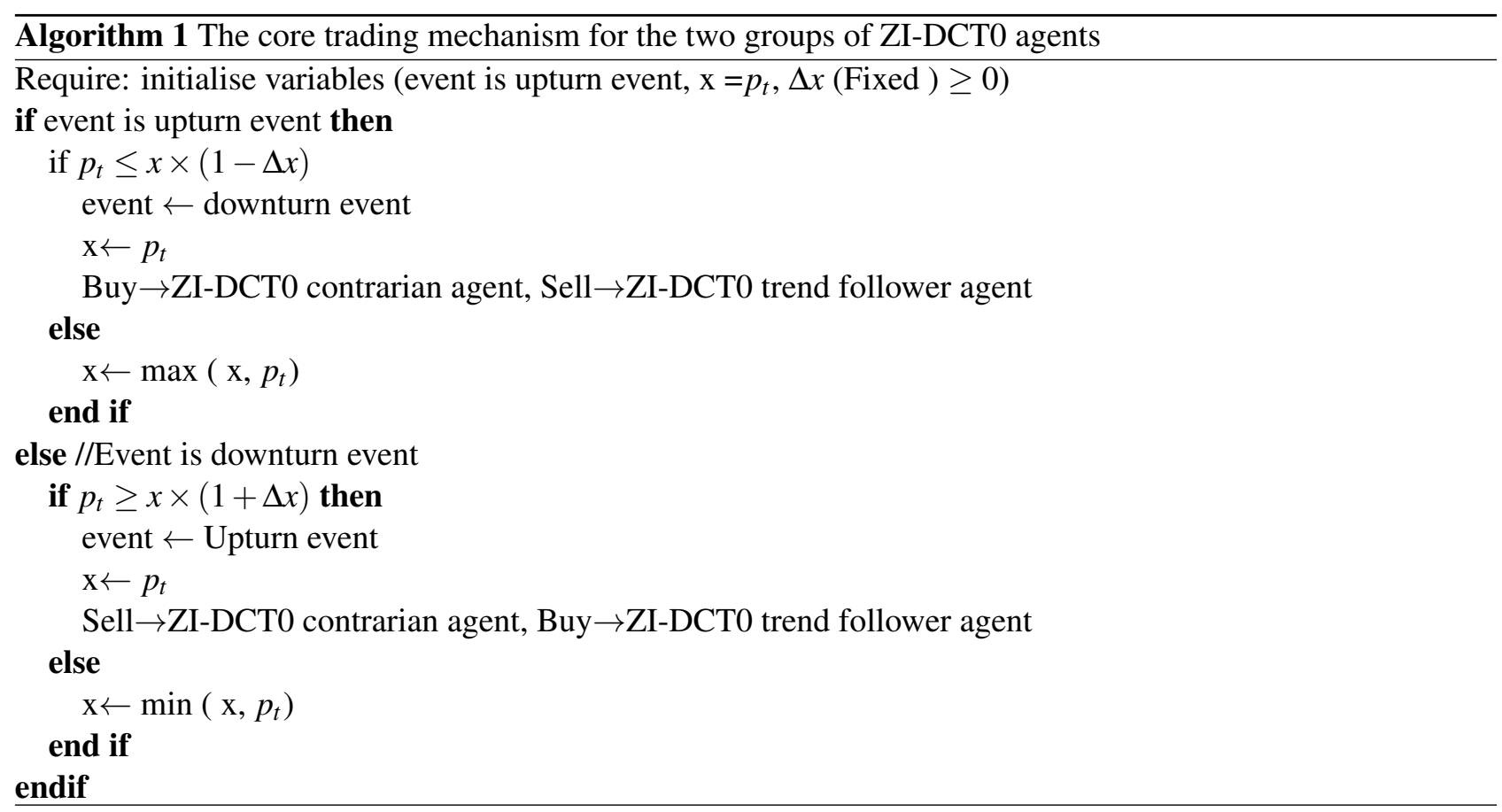

A ZI-DCT0 agent that engages in trend-following behaviour takes advantage of the direction of the price trend movement on the assumption that the current price trend will continue in the same direction. Algorithm 1 illustrates the core trading mechanism for these two groups of ZI-DCT0 agents.

\subsection{GPAs}

Neural networks, genetic algorithms, genetic programming and other machine learning techniques have often been used for financial forecasting. In our work, we chose to use genetic programming (GP) to evolve trading rules for the GP based agents (GPAs) as this technique has been used in related work to model forecasting mechanisms for agents in ABMs Martinez-Jaramillo and Tsang (2009); Serguieva et al. (2011); Chen and Yeh (2001).

The design and behaviour of the GPAs used in this work are based on Martinez-Jaramillo and Tsang (2009). The GPAs are essentially technical traders that use technical analysis in making an investment decision and under certain conditions can vary their trading strategy and switch to fundamental analysis. A GPA trader is able to forecast if the price is going to rise/fall by a defined percentage within a specified number of days. A buy/sell order (an open long/short position) at time $t$ takes place from the prediction of a price rise/drop. A decision to hold will occur when there is no indication of a good opportunity to invest as a result of a price rise or fall. A GPA will use technical analysis for its investment decisions until the difference between the asset price and the fundamental value of the asset price exceeds a defined threshold. Subsequently, the GPA will use fundamental analysis until such a difference between the fundamental value and the asset price disappears.

\subsubsection{The GPA Forecasting Mechanism}

As it is standard with GP, the mechanism starts before the launch of the simulation and each GPA is assigned an initial population of decision rules randomly generated. The decision rules comprise trends following technical indicators. Each individual decision rule is represented by a decision tree which consists of rules and decisions. A rule contains one technical indicator, one relational operator and a threshold value. A single rule cooperates with other rules in the form of one decision tree through logic operators. The logic operators are Not, And, Or and If-Then-Else. The threshold values are the magnitude of the price movement either in terms of a positive or a negative price movement trend. The root node of a decision tree is always an If-Then-Else node. The root node must have three children, the left node of which is a condition node. The two right children could be either a decision node or an If-Then-Else node.

Each decision rule in the initial population has to be evaluated and assigned a fitness value. The fitness function measures the forecasting accuracy for each rule by defining the Rate of Correctness (RC) and the Rate 
of Failure (RF). Given a training set of historical prices over period $T$, the forecasted price of every rule is classified into positive/negative forecasts, where the objective return is realized/not realized. The RC is the number of correct forecasts over the total number of forecasts. The RF is the proportion of forecasts that were wrongly forecasted as being positive, over the number of positive forecasts. The RC and RF are allocated a different weight in the fitness function in order to reflect the preferences of the agents. For example, if a GPA would want to avoid failure, a higher weight for the RF should be used. There are different fitness measures such as the profitability of the investment and the rate of missed opportunities. Li and Tsang in Li and Tsang (2000), study the impact of modifying the fitness measures on the forecasting performance. We have used the RC and RF measures, as they have been used in previous related work Martinez-Jaramillo and Tsang (2009); Serguieva et al. (2011).

A GPA preserves a set of decision rules called a population, Pop, and works through iteration. In each iteration, a new population $(P o p+1)$ is created by updating the fittest decision rules from the last Pop through genetic operators: crossover, mutation and reproduction. Crossover creates a new decision rule by merging two of the fittest decision rules, called parents, from the last Pop. Mutation operates on one decision rule. A random mutation point is selected, the sub-tree rooted at the selected point is deleted, and a new sub-tree is created. Reproduction copies the existing decision rule to the new population. Following the creation of the new $(P o p+1)$, each decision rule in the population will be evaluated on a historical prices training set for the participation in generating a population of decision rules in the next generation. The decision rule with the highest fitness value at the last generation is applied for the GPA's decision to place an order.

After the GPA makes a trade, it re-evaluates its trading rules and consequently increases (decreases) the fitness value for each rule that formed the right (wrong) decision. A GPA is aware of its trading performance in the market and when its wealth falls below a threshold, which is equal to half of its initial wealth, it evolves a new population of rules. The first half of the new population is generated randomly, while the second is generated by retaining half of the GPA's current population of rules. By doing so, the GPA resets the fitness value of each decision rule, and then each rule in the population is evaluated on a historical prices training set. The threshold for the retraining condition is reset to half of the GPA's current wealth. Finally, the GPA uses the decision rule with the highest fitness value with regard to the new population.

The level of GPAs' computational capability is determined by the number of generations and the population size which establish the search space for the GPA to evolve the decision rules. Using a small population limits the search space for a set of investment decision rules and therefore a few and less varied investment decision will be used by a GPA. On the other hand, using a small number of generations means that the evolutionary mechanism has relatively few opportunities to process the search over the set of investment decision rules, which could result in poor rules. For the GPAs used in this study, we chose a population of 50 decision rules, which are evolved over 50 generations, over a training period of 60 days. The computational cost which represents the time for a GPA to evolve good decision rules must not be ignored. Using a large size in terms of both the number of generations and the population size takes a GPA a long time to evolve good decision rules which may not be suitable for investment decisions in high-frequency or other markets Fasli and Kovalchuk (2011). For investment decisions in financial markets, traders have to consider continuous changes in the time series of prices, which sometimes take place to the nearest second.

\subsubsection{Technical Indicators}

Technical analysis uses indicators to forecast price movement and is a significant tool for investment decision making. We have equipped each GPA with six different indicators which have been shown to be useful for forecasting price movements in previous works Martinez-Jaramillo and Tsang (2009); Serguieva et al. (2011).

Given a period of length $L$ and a price time series, the technical indicators are briefly described below while the definitions can be found in Table 1. Each indicator uses just two periods, a short-term and a long-term period, since this is the way they are used by technical analysis traders Martinez-Jaramillo and Tsang (2009). By using these indicators, we sought to limit the size of the search space for a GPA.

The Moving Average (MA) indicator shows the mean price value of an asset over a specific period of time. There are different types of moving average. In this study, the MA is defined by computing the average price of an asset over a specific time.

The Filter indicator (FLR) suggests a buy/sell opportunity depending on whether the price reverses by a predefined amount of size $\Delta x$. For example, if the price movement alters from an upward to a downward trend 


\begin{tabular}{llc}
\hline Technical Indicator & Definition & Period \\
\hline Moving average & $\operatorname{MA}(L, t)=\frac{p(t)-\left(\frac{1}{L} \sum_{i=1}^{L} p(t-i)\right)}{\frac{1}{L} \sum_{i=1}^{L} p(t-i)}$ & $12 \& 50$ days \\
Filter & $F L R(L, t)=\frac{p(t)-\min \{p(t-1), \cdots, p(t-L)\}}{\min \{p(t-1), \cdots, p(t-L)\}}$ & $5 \& 50$ days \\
Trade Break Out & $\operatorname{TRB}(L, t)=\frac{p(t)-\max \{p(t-1), \cdots, p(t-L)\}}{\max \{p(t-1), \cdots, p(t-L)\}}$ & $5 \& 63$ days \\
Volatility & $\operatorname{Vol}(L, t)=\frac{\sigma(p(t), \cdots, p(t-L+1))}{\frac{1}{L} \sum_{i=1}^{L} p(t-i)}$ & $12 \& 50$ days \\
Momentum & $\operatorname{Mom}(L, t)=p(t)-p(t-L)$ & $10 \& 50$ days \\
Momentum Moving Average & $\operatorname{MomMA}(L, t)=\frac{1}{L} \sum_{i=1}^{L} \operatorname{Mom}(L, t-i)$ & $10 \& 50$ days \\
\hline
\end{tabular}

Table 1: The indicators used by GPAs to form decision rules and their respective short and long-term periods.

by $\Delta x$ from the highest price, then the FLR shows a sell opportunity.

Technical analysis indicates that an upward (downward) price trend is likely to halt (reserve) at a resistance (support) point, which is the point at which the price trend stops moving upwards (downwards). But it is possible that the price trend will continue in the same direction when it breaks through the resistance/support points. The Trade Break Out (TRB) indicator indicates a buy (sell) opportunity when an asset's price moves above (below) its point of resistance (support).

The volatility indicator ( $\mathrm{Vol}$ ) indicates a buy opportunity when there is a period of declining volatility in the price time series; this would indicate an upward trend. A period of increasing volatility will possibly indicate a sharp downward trend which is an indication of a sell opportunity.

The Momentum (Mom) indicator measures the rate at which an asset's price is changing. When the momentum is positive (negative), it indicates an upward (downward) trend. Following from this, we can then use the Mom indicator to calculate its moving average (MomMA).

\subsubsection{Fundamental Analysis}

The fundamental analysis of an asset price is dependent on generating a fundamental value based on analysing the factors which could possibly influence the changes of the asset price such as financial market reports, earnings and sales on the asset price. The trader then compares the fundamental value with the current asset price to make an investment decision. Hence, the performance of a decision based on fundamental analysis depends on the precision of generating the fundamental value. In the literature, there are different ways for defining the fundamental value of an asset price for investment decision making. For example, in Farmer (1998) there is one fundamental value for all the agents in the market, whereas in LiCalzi and Pellizzari (2003) agents possess different fundamental values.

In our work, the GPAs are able to vary their trading strategy using fundamental analysis. A GPA will use technical analysis for its investment decisions until the difference between the asset price and the fundamental value of the asset price exceeds a threshold defined by the GPA. Afterwards, the GPA will use fundamental analysis until such a difference between the fundamental value and the asset price disappears. The GPAs in the market possess one fundamental value of the asset price just as in Farmer (1998). The reason behind using just one fundamental value is to avoid complexity in terms of the GPAs' trading behaviour. At time $t$, the fundamental value of the asset price $f_{t}$ is given by an exogenous random process and is defined as $f_{t}=f_{t-1}+\eta_{t}$, where $\eta_{t}$ is a normal noise process with mean $\mu$ and standard deviation $\sigma$.

Although GPAs possess one fundamental value, not all GPAs will be making the same decision. This is because GPAs that adopt fundamental analysis for their strategy are committed to a fixed threshold which is generated randomly from uniform intervals before the launch of the market. A GPA takes a decision to invest if the asset price departs from the fundamental value by an amount equal to, above, or below the threshold. The GPAs will continue updating their beliefs with regard to the market until the difference between the asset 
price and the fundamental value is lower than a threshold. This fundamental behaviour was introduced in Martinez-Jaramillo and Tsang (2009).

\section{Experimental Results}

In this section, we report on the experiments undertaken in the ABFXM that we developed and the three strategies. Our aim in particular, is to examine the impact of the use of the three strategies in the reproduction of the stylized facts in the FX market; this can subsequently inform the design of trading strategies and decision support systems for the FX market.

\subsection{Overview of the FX Market}

The Foreign Exchange (FX) market is considered the largest, most liquid and efficient financial market in the world Dacorogna et al. (2001). The FX market is where the buying, selling and exchanging of currencies takes place. It is important to highlight that the FX market is not a single market, but is composed of a global networks of FX markets that connect investors from all around the world. The fact that the FX market is a decentralized market means that there is no central marketplace, and that transactions are conducted over the counter. Traders in the FX market include governments, central banks, large banks, retail investors, institutional investors, etc.

With the advent of retail market-maker FX market online platforms, individual retail traders represent an important growth part of the FX market. Most FX trading firms are market-makers. A market-maker is a company which provides liquidity for a particular currency pair, and quotes both a buy and a sell price for such a currency pair on its platform. The market-maker buys from and sells to its clients and other market-makers, to make a profit on the bid-offer spread or return. In other words, the market-maker takes the opposite side of a trade and earns its commission from the difference between the bid and the offer price.

\subsection{Datasets}

In this study, we used two high-frequency historical datasets, made available to us by OANDA Corporation ${ }^{3}$, which provides an online foreign currencies trading platform. We use a historical dataset which contains data samples of EUR/USD prices between the period January 1, 2007 to March 5, 2009. Each record in the dataset comprises three fields: (a) a bid and (b) an ask price of a currency pair at (c) a timestamp. This dataset is fed into the ABFXM.

The second dataset is a unique high-frequency dataset of individual traders' historical transactions at an account level, made available on an anonymous basis spanning 2.25 years, from 1/1/2007 to 5/3/2009. OANDA trading platform stores the historical details of individual traders' transactions over a long time horizon. This dataset contains about 147 million historical transactions carried out by 45,845 different accounts, trading in 48 different currency pairs under the same terms and conditions. Each historical transaction record includes: the transaction type, the timestamp, the traded currency pair, the execution price, the number of traded units, and the amount traded. We used this second dataset as explained in Aloud et al. (2012), to establish stylized facts of the transactions data in the high-frequency FX market. Stylized facts (i.e. statistical patterns in financial data) can serve as a benchmark for evaluating the behaviour and data that are generated from ABMs and enable conclusions to be drawn regarding the ability of the ABM to model the real market. However, identifying stylized facts is not straightforward: to be able to do this, one needs to have access to large volumes of real data. Fortunately, our study was provided with such data. A brief description of the stylized facts of the transactions data established is provided here, for more details we refer the reader to Aloud et al. (2012). The established stylized facts can be grouped under three main headings: seasonality, correlation behaviour and scaling laws ${ }^{4}$.

\footnotetext{
${ }^{3}$ http://www.oanda.com/

${ }^{4}$ Please note that the results from the analysis of the FX market data are illustrated side by side with the results of the ABFXM so that direct comparisons can be drawn.
} 


\subsection{Results}

In this section, we report the stylized facts of FX market trading activity established (a) in the real market data in comparison to those established from the ABFXM populated with: (b) ZI-CV agents; (c) ZI-DCT0 agents; (d) GPAs; a mixture of (e) 50\% ZI-CV and 50\% ZI-DCT0 agents; (f) 70\% ZI-CV and 30\% ZI-DCT0 agents; (g) 30\% ZI-CV and 70\% ZI-DCT0 agents; (h) ZI-DCT0 and GPAs where GPAs <50\%; (i) ZI-DCT0 and GPAs where GPAs $\geq 50 \%$; (j) ZI-CV and GPAs where GPAs $<50 \%$ and finally (k) ZI-CV and GPAs where GPAs $\geq 50 \%$. Our aim in populating the ABFXM with different combinations of the three trading strategies, is to examine the impact of such combinations in the reproduction of stylized facts in the FX market transaction data and whether a significant proportion of one strategy over another has an effect on the dynamics of the market and the emergence of the stylized facts.

The simulation uses historical bid and ask prices for the EUR/USD currency pair over a defined one month period by feeding these prices into the market and having the agents respond to the price changes. We use this dataset as our purpose is to explore the impact of various strategies on the transactions data generated within the ABFXM. The price data are used to anchor the simulation results so that we can establish whether the transactions data generated by the ABFXM have the same characteristics and properties as the real market data. The historical dataset of prices acts as a constant so that multiple simulation runs can be performed in which different strategies and their impact can be systematically studied without having to worry about price setting in the market. This enables us to study the ensuing behaviour and data generated from the market against those of the real market and also compare different settings against one another.

The agent population in the ABFXM is $10^{4}$. The only exception is case (d) where the ABFXM is populated just by GPAs and due to limited computational capacity there are 8,000 GPAs running. The stylized facts generated from the ABFXM are averaged over 30 independent simulation runs, each run being one simulated month, and with different initial seeds provided by random number generators, and different time horizons over the 2.25 years of the EUR/USD prices data samples. We performed the different independent simulation runs with the same parameter configuration values but with different seeds and time horizons to ensure that the results of the simulation are consistent; this allows to establish the robustness and accuracy of the simulation results.

\subsubsection{Stylized Facts}

\section{Seasonality}

The FX market trading activity data exhibit periodic patterns called seasonality which have been confirmed in studies such as Dacorogna et al. (2001); Ito and Hashimoto (2006). The intraday seasonality relates quantities of the market trading activities to the time of day when these quantities are observed. More specifically, an intraday analysis constructs a uniform time grid with 24 hourly intervals for the statistical analysis of the different market trading activities such as with regard to trade number and volumes. The shape of the FX market intraday seasonality is referred to as a double U-shape or Camel shape because of the two peaks, in which the second peak is higher than the first. In detail, the intensity of trading activity starts to peak within the opening business hours of the main FX market centres in the morning, declines during the lunch break and peaks in the afternoon. Outside the business hours, the intensity of trading activity gradually declines. The analysis of our dataset has confirmed the same result Aloud et al. (2012). We measure the precision of the seasonality statistical results using a paired t-test. Given the seasonality statistical results of the different FX market trading activities, it can be determined with a 95\% confidence level, whether or not the means of the seasonal statistical data from the real FX market data and the ABFXM data are significantly the same. If the mean values of the statistical data differ significantly, this provides evidence that the collective trading behaviour of the agents in the ABFXM does not resemble the trading activity in the FX market.

Figure 1 shows the intraday seasonality of the trade numbers from the different data samples as explained in section 5.3 with case (a) being the seasonality exhibited in the real data. From Figure 1, the intraday seasonality of the trade numbers exhibits the double U-shape for two cases when the ABFXM is populated with (c) just ZI-DCT0 and (h) a mixture of ZI-DCT0 and GPAs $(<50 \%)$. In contrast, when the ABFXM is populated with GPAs $(\geqslant 50 \%)$, the U-shape pattern does not emerge. Similarly, all other cases do not exhibit this intraday seasonality measure. In particular, in the three cases $(b, j$ and $k$ ) where the ABFXM is populated with ZI-CV agents, the intensity of trade numbers increases sharply around 4:00 am. Afterwards, the intensity of trade 


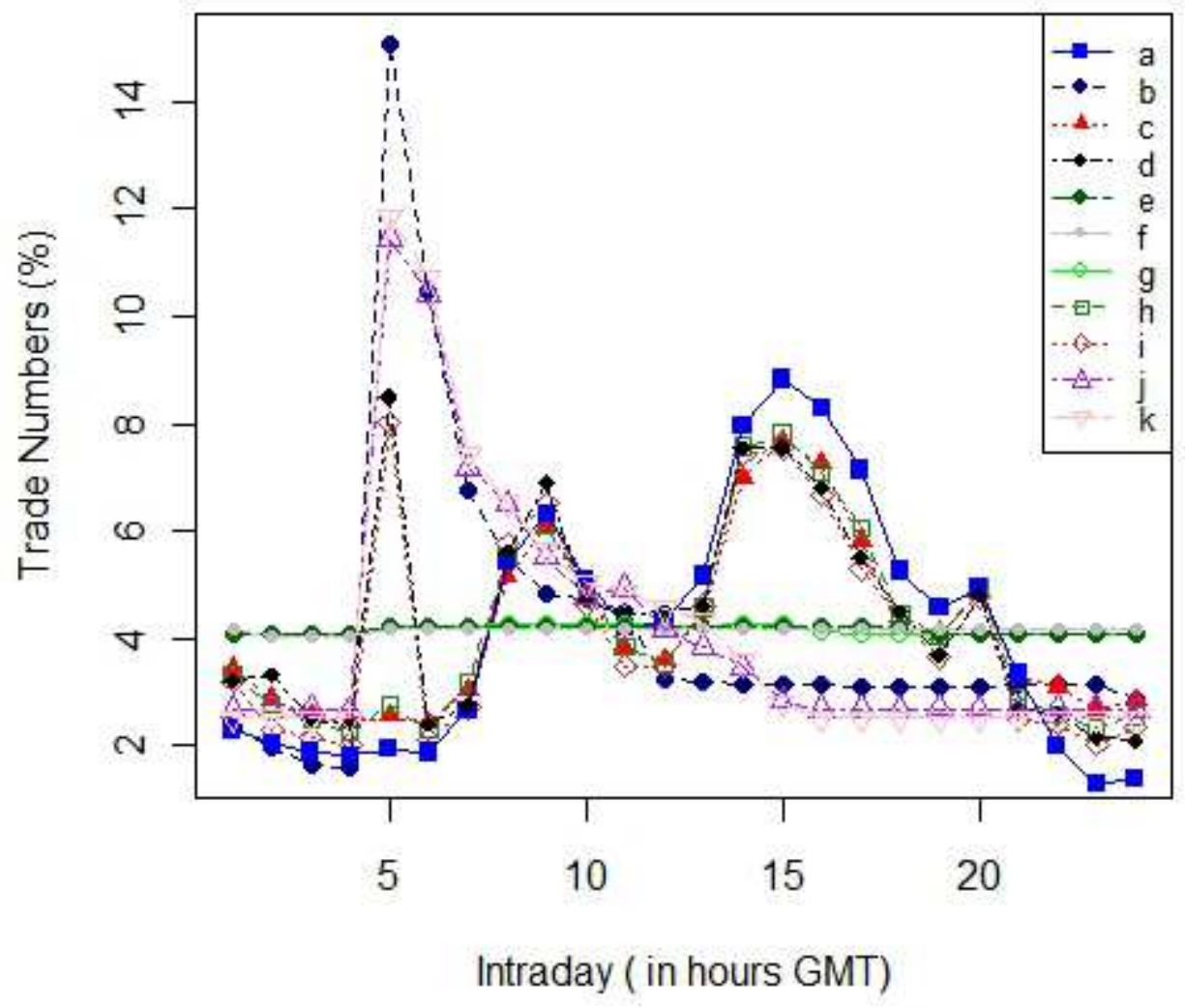

Figure 1: The intraday seasonality of the trade numbers from (a) the dataset of EUR/USD transactions, the ABFXM populated with (b) ZI-CV, (c) ZI-DCT0, (d) GPAs, (e) 50\% ZI-CV and 50\% ZI-DCT0 , (f) 70\% ZICV and 30\% ZI-DCT0, (g) 30\% ZI-CV and 70\% ZI-DCT0, (h) ZI-DCT0 and GPAs (<50\%), (i) ZI-DCT0 and GPAs ( $\geqslant 50 \%)$, (j) ZI-CV and GPAs $(<50 \%)$, (K) ZI-CV and GPAs $(\geqslant 50 \%)$. 


\begin{tabular}{|c|c|c|c|c|c|c|c|c|}
\hline & \multicolumn{2}{|c|}{ (b) } & \multicolumn{2}{|c|}{ (c) } & \multicolumn{2}{|c|}{ (d) } & \multicolumn{2}{|c|}{ (e) } \\
\hline & $t s v$ & corr & $t s v$ & corr & $t s v$ & corr & $t s v$ & corr \\
\hline Trade numbers & $2.2 \mathrm{E}-10$ & -0.21 & $-7.1 \mathrm{E}-10$ & +0.98 & $-3.9 \mathrm{E}-02$ & +0.68 & $-1.3 \mathrm{E}-10$ & +0.52 \\
\hline Trade volumes & $2.2 \mathrm{E}-11$ & -0.23 & 4.6E-16 & +0.87 & 4.7E-02 & +0.50 & $-7 E-10$ & +0.99 \\
\hline Opening positions & $-2.7 \mathrm{E}-10$ & -0.21 & $-2.1 \mathrm{E}-09$ & +0.98 & $-5.3 \mathrm{E}-02$ & +0.66 & $-1.3 \mathrm{E}-10$ & +0.52 \\
\hline \multirow[t]{2}{*}{ Closing positions } & $5.3 \mathrm{E}-10$ & -0.20 & 2.3E-09 & +0.98 & $-1.7 \mathrm{E}-02$ & +0.78 & $1.3 \mathrm{E}-10$ & +0.53 \\
\hline & \multicolumn{2}{|c|}{ (f) } & \multicolumn{2}{|c|}{$(\mathrm{g})$} & \multicolumn{2}{|c|}{ (h) } & \multicolumn{2}{|c|}{ (i) } \\
\hline Trade numbers & $-1.3 \mathrm{E}-10$ & +0.40 & $-2.6 \mathrm{E}-10$ & +0.32 & $-3.2 \mathrm{E}-09$ & +0.99 & $-3.7 \mathrm{E}-02$ & +0.78 \\
\hline Trade volumes & $-1.5 \mathrm{E}-09$ & +0.99 & $-1.5 \mathrm{E}-09$ & +0.99 & $-1.4 \mathrm{E}-09$ & +0.99 & $5.8 \mathrm{E}-02$ & +0.55 \\
\hline Opening positions & $5.8 \mathrm{E}-17$ & +0.39 & $-3.9 \mathrm{E}-10$ & +0.30 & $-3.8 \mathrm{E}-16$ & +0.99 & $-5.2 \mathrm{E}-02$ & +0.70 \\
\hline \multirow[t]{2}{*}{ Closing positions } & $2.5 \mathrm{E}-10$ & +0.40 & $3.8 \mathrm{E}-10$ & +0.33 & $1.5 \mathrm{E}-09$ & +0.99 & $-1.3 \mathrm{E}-02$ & +0.85 \\
\hline & \multicolumn{2}{|c|}{ (j) } & \multicolumn{2}{|c|}{ (k) } & & & & \\
\hline Trade numbers & $-3.63 \mathrm{E}-16$ & -0.23 & $-2.42 \mathrm{E}-10$ & -0.22 & & & & \\
\hline Trade volumes & $-1.90 \mathrm{E}-10$ & +0.05 & $-3.75 \mathrm{E}-11$ & -0.23 & & & & \\
\hline Opening positions & $-1.89 \mathrm{E}-10$ & -0.24 & $-1.21 \mathrm{E}-10$ & -0.23 & & & & \\
\hline Closing positions & $2.52 \mathrm{E}-10$ & -0.23 & $3.03 \mathrm{E}-10$ & -0.20 & & & & \\
\hline
\end{tabular}

Table 2: T-statistic and correlation when the ABFXM is populated with (b) ZI-CV, (c) ZI-DCT0, (d) GPAs, (e) 50\% ZI-CV and 50\% ZI-DCT0, (f) 70\% ZI-CV and 30\% ZI-DCT0, (g) 30\% ZI-CV and 70\% ZI-DCT0, (h) ZI-DCT0 and GPAs $<50 \%$, (i) ZI-DCT0 and GPAs $\geqslant 50 \%$, (j) ZI-CV and GPAs $<50 \%$, (K) ZI-CV and GPAs $\geqslant 50 \%$.

numbers declines sharply, and then remains very stable throughout the rest of the day, in contrast to the real market. In all of the three cases where the ABFXM is populated with a mixture of ZI-CV and ZI-DCT0 agents, the intensity of trade numbers remains stable throughout the day.

From Table 2 and according to the paired t-tests with a 95\% confidence level linked to the hypothesis $\left(\mu_{R}-\mu_{S}=0\right)$, the seasonality statistics are exhibited in all the different cases. However, the closer correlation results are clearly exhibited by cases (c) and (h), whereas the correlations in the other cases are not satisfactory. In (b), where the ABFXM comprises just ZI-CV agents, the correlations between the seasonality statistics of real data and the simulation data are negative. In (e,f,g), where the ABFXM comprises a mixture of ZI-CV and ZI-DCT0 agents, the Pearson correlation values are low for the trade numbers, while very strong - close to 1 - for the trade volumes. Such results are of concern as the empirical works in the literature indicate that there is a strong correlation between trade numbers and volumes Dacorogna et al. (2001); Ito and Hashimoto (2006). In the two cases (j,k), where the ABFXM consists of a mixture of ZI-CV agents and GPAs, the Pearson correlation values are negative for the trade numbers, and very low for the trade volumes where GPAs $<50 \%$.

The periodic patterns are correlated with the changes in the behaviour of the worldwide FX market participants Dacorogna et al. (2001). We believe that the lack of a trading strategy in the ZI-CV agents, may be responsible for the absence of such periodic patterns. The randomness in the ZI-CV agents' behaviour also makes it difficult to interpret the incompatible results between the correlation values for trade numbers and volumes.

Although, the GPA models in our experiments had been calibrated, such strategies do not seem to yield results close to those of real data. Identifying which of the parameters in the GPAs' strategy are responsible for the deviation from the periodic patterns observed in real data is not straightforward due to the underlying complexity of the strategy. The experimental results suggest that a random strategy (even with budget constraints) or a strategy that involves learning is not able to reproduce the stylized facts of the FX market to a satisfactory extent. The ZI-DTC0 strategy on the other hand which is based on the idea of identifying periodic patterns and responding to them, seems to achieve results that are close to those of the real market.

\section{Correlation Behaviour}

The correlation stylized facts relate to the positive linear relationship that was found between trade numbers and volumes, numbers of buy and sell executed orders, number of opening and closing positions, and intraday price volatilities and trade numbers and volumes Aloud et al. (2012).

The correlation between the different trading activities in the FX market data (Table 3 (a)), reveals a positive 


\begin{tabular}{lcccccc}
\hline & $(\mathrm{a})$ & $(\mathrm{b})$ & $(\mathrm{c})$ & $(\mathrm{d})$ & $(\mathrm{e})$ & $(\mathrm{f})$ \\
\hline Trade numbers and volumes & +0.81 & +0.70 & +0.92 & +0.86 & +0.04 & +0.02 \\
Buy and sell orders & +0.95 & +1 & +0.99 & +0.85 & +1 & +1 \\
Opening and closing positions & +0.98 & +1 & +0.98 & +0.80 & +1 & +1 \\
Volatilities and trades & +0.43 & -0.10 & +0.46 & +0.05 & -0.03 & +0.03 \\
Volatilities and volumes & +0.28 & -0.06 & +0.31 & -0.01 & -0.09 & -0.03 \\
\hline \multicolumn{1}{c}{$(\mathrm{g})$} & $(\mathrm{h})$ & $(\mathrm{i})$ & $(\mathrm{j})$ & $(\mathrm{k})$ & \\
\hline Trade numbers and volumes & +0.04 & +0.88 & +0.89 & +0.41 & +0.59 & \\
Buy and sell orders & +1 & +1 & +0.89 & +1 & +1 & \\
Opening and closing positions & +1 & +0.96 & +0.83 & +1 & +1 & \\
Volatilities and trades & +0.01 & +0.01 & +0.03 & -0.12 & -0.12 & \\
Volatilities and volumes & -0.03 & -0.04 & -0.01 & +0.02 & -0.01 & \\
\hline
\end{tabular}

Table 3: Correlation coefficients computed for the different EUR/USD trading activity from (a) the dataset of EUR/USD transactions, the ABFXM populated with (b) ZI-CV, (c) ZI-DCT0, (d) GPAs, (e) 50\% ZI-CV and 50\% ZI-DCT0, (f) 70\% ZI-CV and 30\% ZI-DCT0, (g) 30\% ZI-CV and 70\% ZI-DCT0, (h) ZI-DCT0 and GPAs $(<50 \%)$, (i) ZI-DCT0 and GPAs ( $\geqslant 50 \%)$, (j) ZI-CV and GPAs ( $<50 \%)$, (K) ZI-CV and GPAs $(\geqslant 50 \%)$.

linear relationship between trade numbers and volumes, numbers of buy and sell executed orders, number of opening and closing positions, and intraday price volatilities and trade numbers and volumes. From Table 3 (c), we can see that the results when the ABFXM is populated with only ZI-DCT0 agents, case (c), exhibit very similar correlation behaviour to that of the real data. This means that there are linear dependencies between the current and past values of the different trading activities in the FX market data. We believe that the success of the ZI-DCT0 approach in modelling agents is the link between the variations in price changes and thresholds of different sizes which trigger the agents to trade.

In contrast, in all other cases the correlation is not satisfactory. These results indicate that there is no dependency between the variations in price changes and the trading activity emerging from the ABFXM with ZI-CVs or GPAs. The ZI-CV agents trade randomly, subject to budget constraints, and therefore do not respond to price changes. On the other hand, GPAs use different forms of technical and fundamental analysis of price changes for their investment decisions, as described in section 4.3. Hence, this results in a variety of decision rules used by the GPAs. When the ABFXM is populated with a mixture of ZI-CV and ZI-DCT0 agents, cases $(\mathrm{e}, \mathrm{f}, \mathrm{g})$, this affects extensively the correlation between the trade numbers and volumes. The statistics show that there is weak relationship between trade numbers and volumes which is contrary to what has been confirmed using real market data Dacorogna et al. (2001). Tracking the ZI-CV agents' trading activity, we found that a low level of cash in their accounts affects the order size of their trade, which consequently results in a low level of trade volumes in the market. The low cash in the ZI-CV agents' accounts stems from their unsuccessful investments during the simulation run. This explains why there is lack of autocorrelation between the ZI-CV agents' trade numbers and volumes.

\section{Scaling Laws}

Scaling laws establish quantitative relationships between the size of price movements and the different market trading activities as a function of the time interval at which market trading activity is measured. To define scaling laws for the EUR/USD trading activity, and using the directional-change event approach, we identify directional-change (DC) and overshoot (OS) events in the EUR/USD price time series for thresholds of different sizes, ranging from $0.10 \%$ to $0.80 \%$. Given a fixed percentage threshold, the average observed (a) trade numbers, (b) trade volumes, number of (c) opening and (d) closing positions observed during a DC and an OS event, is scale-invariant to the size of this threshold Aloud et al. (2012). Our study has also uncovered six quantitative relationships amongst the four scaling laws, holding across EUR/USD trading activity. These six quantitative relationships specify that, on average, an OS event contains roughly twice as many trade numbers and volumes, and numbers of opening and closing positions, as a DC event. In addition, an OS event contains approximately the same number of opening and closing positions, while similar features hold for a DC event. We examine the exhibition of the scaling laws by defining the linear dependence. The adjusted $R^{2}$ value estimates the significance of fit of the line. The adjusted $R^{2}$ value can take any value less than or equal to 1 , with 
a value closer to 1 signifying a better fit. Consequently, the closer the points to the line, the better the fit. It is important to highlight that the adjusted $R^{2}$ value is not sensitive to the number of observed points within the data sample.

The scaling laws as confirmed in the FX market data are plotted in Figure 2 (a). Table 4 (a) reports the adjusted $\mathrm{R}^{2}$ values and standard errors of the fits. The existence of the six quantitative relationships amongst the four scaling laws can also be established from the same figure.

When the ABFXM is populated with just ZI-CVs, case (b), it is not possible to discern the scaling laws and the dynamics of the trading activity do not match those observed in the real data. Hence, the graphs could not be drawn and this case has been omitted from Figure 2 and Table 4 . This is because the intensity of trading activity increases sharply during the few first hours of the simulation, after which the trading activity declines suddenly and sharply to a much lower level, and continues to be stable at this very low level during the remaining time of the simulation (Figure 1). This could be due to the ZI-CV agents' random strategy and as a consequence their inability to react to price changes in the market.

The scaling laws and the six quantitative relationships amongst them, are exhibited in the data generated from the ABFXM populated with just ZI-DCT0 agents, Figure 2 (c). Table 4 (c) reports the adjusted $\mathrm{R}^{2}$ values and standard errors of the fits. The significance of the fit of the line which is an indication of linear dependency between the different sizes of the price movements and the observed market trading activity measured at these different sizes of price movements, is obvious from the adjusted $\mathrm{R}^{2}$ values. The standard errors of the fits are roughly equivalent to the estimated errors for scaling laws in the empirical data reported in Table 4 (a). We therefore conclude that a key element in modelling the agents' strategies is the linking of variations in price changes to thresholds of different sizes in order to trigger trading behaviour on the part of the agents.

In the three cases (e, f, g), where the ABFXM is populated with a mixture of ZI-CV and ZI-DCT0 agents, surprisingly the simulation results exhibit the scaling laws according to the adjusted $\mathrm{R}^{2}$ values, Table 4 (e, $\mathrm{f}, \mathrm{g}$ ). However, the six quantitative relationships amongst the scaling laws are not exhibited, as shown from Figure 2 (e) and 3 (f, g). Additionally, the trade numbers are much higher than in the real FX market data, as can be seeing from Figure 2 (a). Such results are questionable, in that unrealistically high trade numbers occur during DC and OS events. One possible reason is that the ZI-CVs do not react to price changes in the market, and consequently do not consider a DC event as a pattern set up for the price trend movement. Such a pattern influences agents' decisions, assuming that the current price trend will continue in the same direction or move in the opposite direction. Therefore, this might explain why the DC and OS events contain roughly the same number of trades in the ABFXM populated with ZI-CVs. This is in contrast to the real FX market data wherein an OS event contains twice as many trades as a DC event.

The scaling laws and the quantitative relationships amongst them are not exhibited in the data produced from the ABFXM populated with just GPAs or a mixture of ZI-DCT0 and GPAs, Figure 2 (d) and 3 (h, i). From Table $4(\mathrm{~d}, \mathrm{~h}, \mathrm{i})$, the adjusted $\mathrm{R}^{2}$ values are not very close to 1 , which implies non-significant fit. The scaling laws are exhibited under DC events from the ABFXM populated with a mixture of ZI-CV and GPAs where GPAs $<50 \%$ (Figure 4 (j)). The adjusted $R^{2}$ values in Table 4 (j) show the significance of the fit. However, the scaling laws of trade numbers are not exhibited under OS events from the ABFXM populated with a mixture of ZI-CV and GPAs where GPAs $<50 \%$. The adjusted $\mathrm{R}^{2}$ values in Table $4(\mathrm{k})$ are negative. In the case of the ABFXM populated with a mixture of ZI-CV and GPAs where GPAs $\geqslant 50 \%$ (Figure $4(\mathrm{k})$ ), the scaling laws are not exhibited. According to the results, the ensuing GPAs' behaviour in the ABFXM does not reproduce the scaling laws, thus supporting other studies which emphasize that some properties in the financial markets may not indeed be the result of interactions between agents who possess sophisticated learning and adaptation behaviour Alfi et al. (2009); Daniel (2006); Tseng et al. (2010).

\section{Return on Investment (ROI)}

ROI is a performance measure which is defined as the total return with regard to an agent's investment over a defined period, divided by the cost of the investment. The ROI is expressed as a percentage and can either be positive or negative, which means respectively that the agent achieves a profit or makes a loss. We measure the ROI for each agent's account over a period of a simulated month. The aim is to evaluate the profitability and performance of the agents' trading strategies in the ABFXM. Table 5 shows a number of basic descriptive statistics of the agents' ROI from different simulation runs.

For the situation in which the ABFXM is populated with ZI-CV agents, case (b), the performance of such 
Trade Numbers (a)

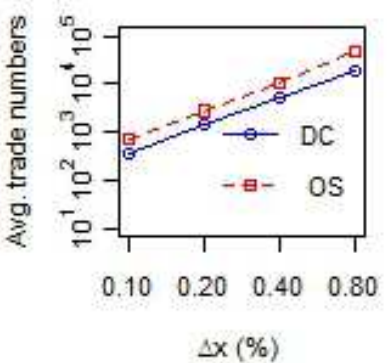

Trade Numbers (c)

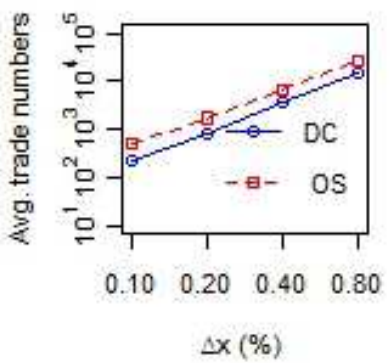

Trade Numbers (d)

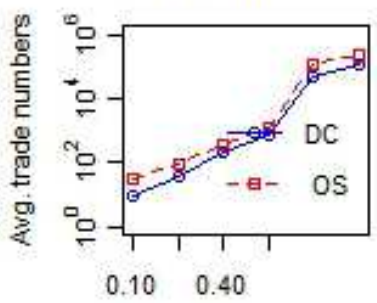

$\Delta x(\%)$

Trade Numbers (e)

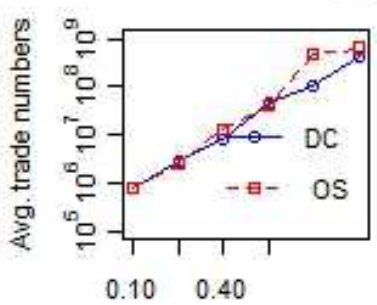

$\Delta x(\%)$

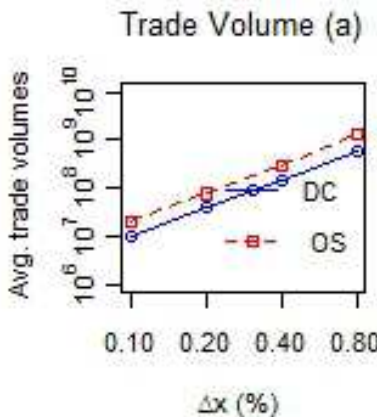

Trade Volumes (c)

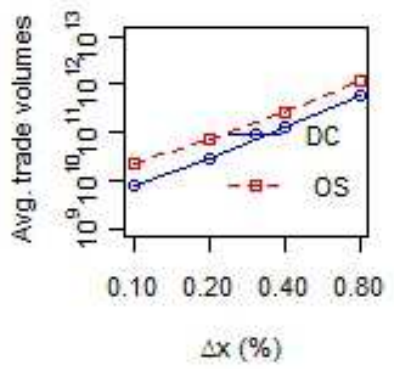

Trade Volumes (d)

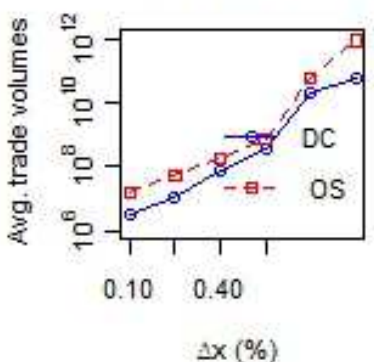

Trade Volumes (e)

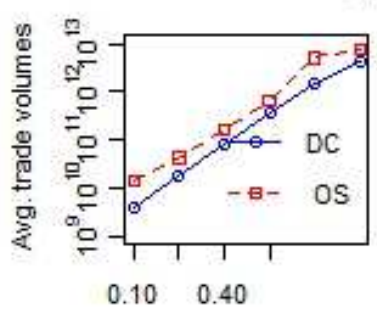

$\Delta x(\%)$
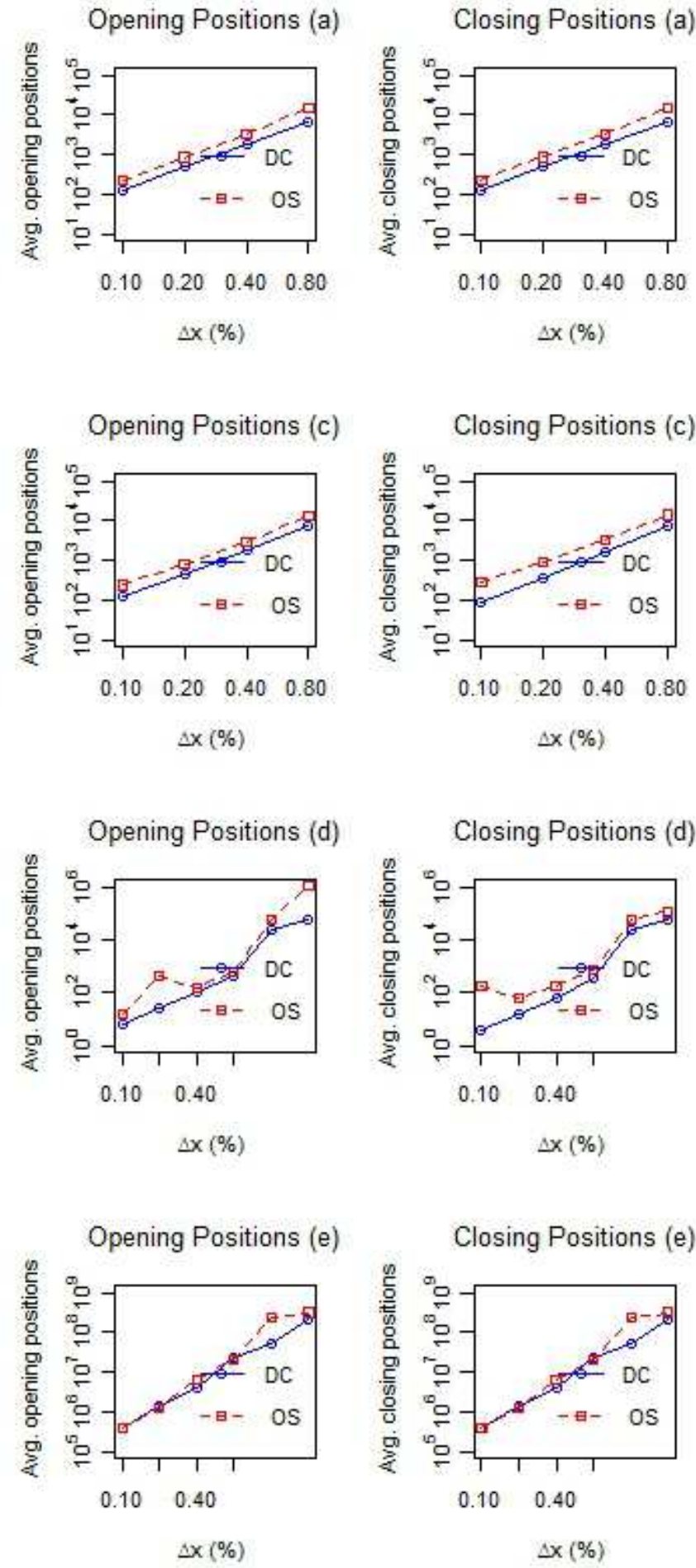

Figure 2: Scaling laws are plotted where the $\mathrm{x}$-axis shows the price moves thresholds of the EUR/USD observations and the y-axis shows the average: trade numbers; trade volumes; number of opening positions; and number of closing positions. Scaling laws are defined from (a) the dataset of EUR/USD transactions, the ABFXM populated with (c) ZI-DCT0, (d) GPAs, (e) 50\% ZI-CV and 50\% ZI-DCT0. 
Trade Numbers (f)

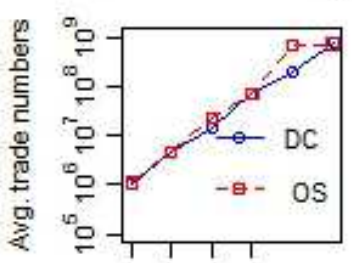

$0.10 \quad 0.40$

$\Delta \times(\%)$

Trade Numbers $(\mathrm{g})$

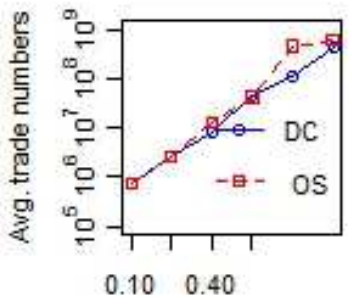

$\Delta x(\%)$

Trade Numbers (h)

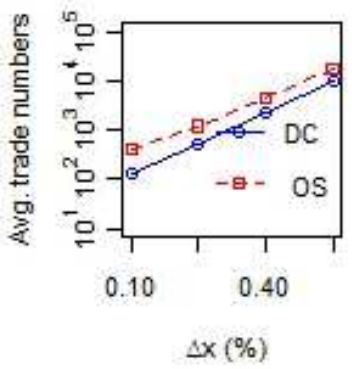

Trade Numbers (i)

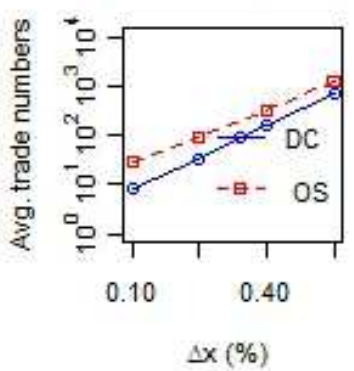

Trade Volumes (f)

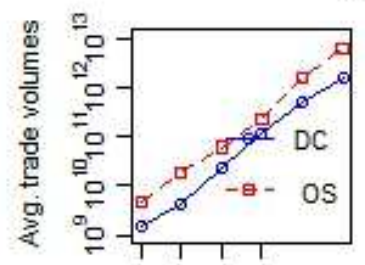

$0.10 \quad 0.40$

$\Delta x(\%)$

Trade Volumes $(\mathrm{g})$

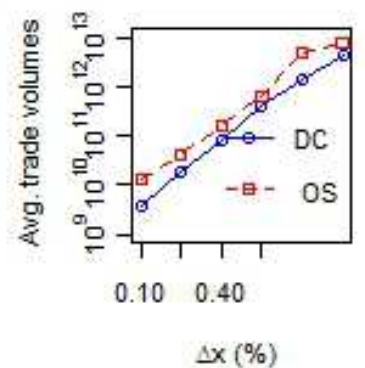

Trade Volumes (h)

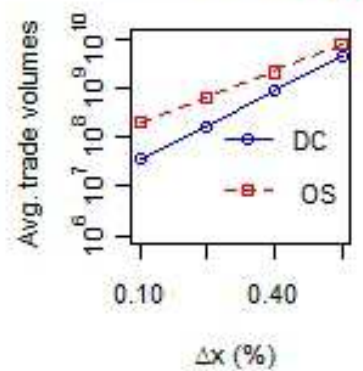

Trade Volumes (i)

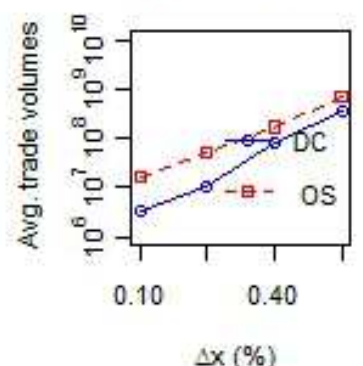

Opening Positions ( $f$ )

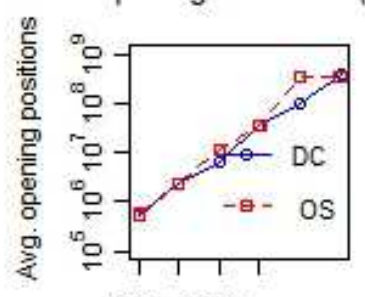

$0.10 \quad 0.40$

$\Delta x(\%)$

Opening Positions (g)

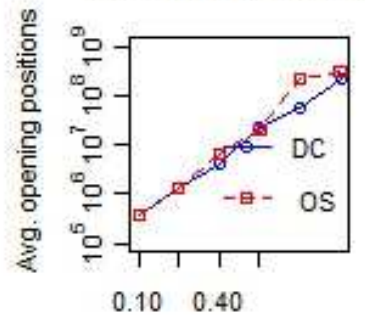

$\Delta x(\%)$

Opening Positions (h)

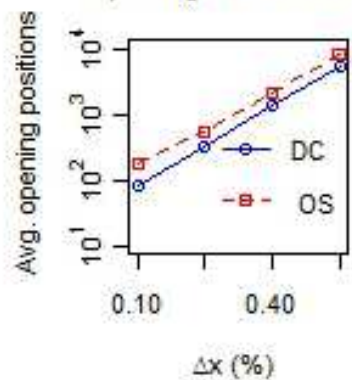

Opening Positions (i)

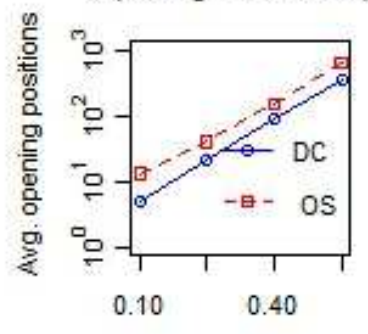

$\Delta x(\%)$
Closing Positions (f)

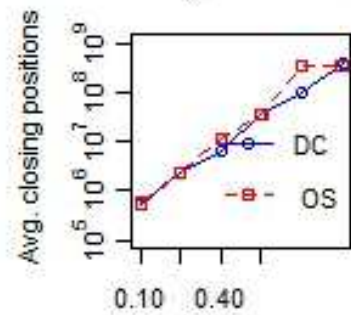

$\Delta \times(\%)$

Closing Positions (g)

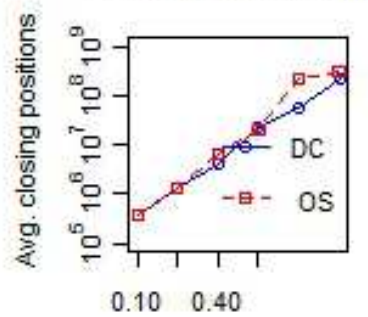

$\Delta x(\%)$

Closing Positions (h)

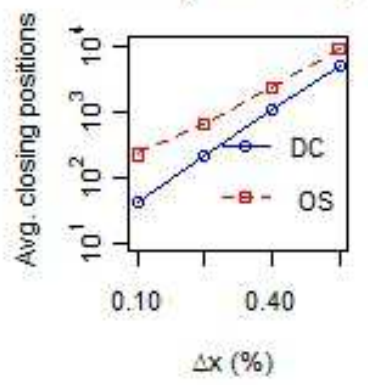

Closing Positions (i)

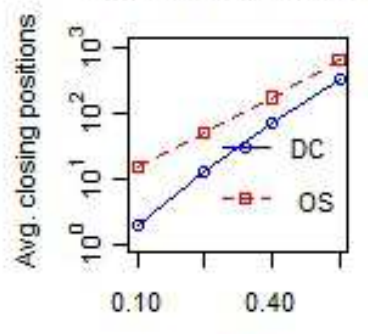

$\Delta x(\%)$

Figure 3: Scaling laws are plotted where the $\mathrm{x}$-axis shows the price moves thresholds of the EUR/USD observations and the $y$-axis shows the average: trade numbers; trade volumes; number of opening positions; and number of closing positions. Scaling laws are defined from (f) 70\% ZI-CV and 30\% ZI-DCT0, (g) 30\% ZI-CV and 70\% ZI-DCT0, (h) ZI-DCT0 and GPAs $(<50 \%)$ and (i) ZI-DCT0 and GPAs ( $\geqslant 50 \%)$. 

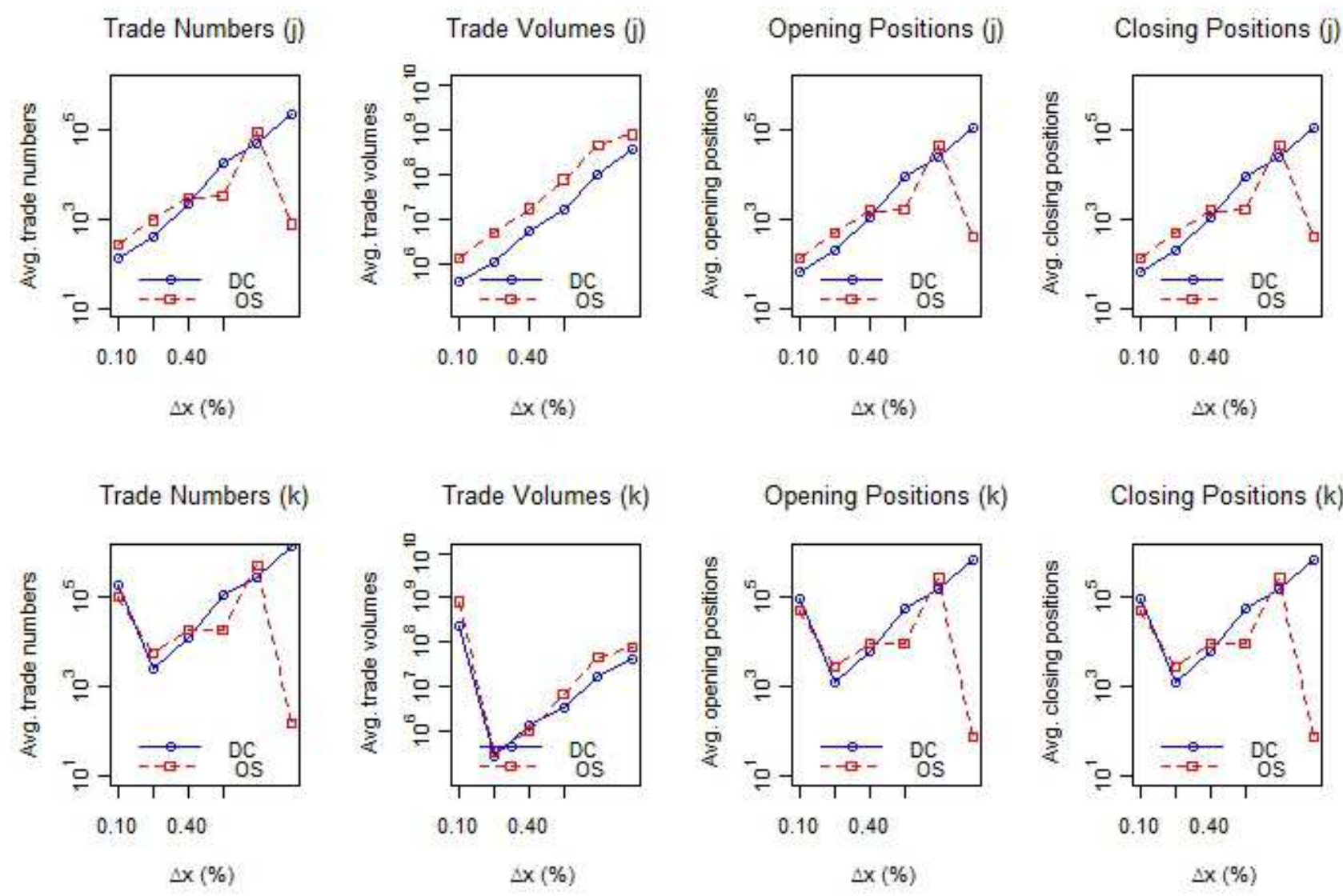

Figure 4: Scaling laws are plotted where the $\mathrm{x}$-axis shows the price moves thresholds of the EUR/USD observations and the $y$-axis shows the average: trade numbers; trade volumes; number of opening positions; and number of closing positions. Scaling laws are defined from (j) ZI-CV and GPAs ( $<50 \%)$, (K) ZI-CV and GPAs $(\geqslant 50 \%)$. 


\begin{tabular}{|c|c|c|c|c|c|c|c|c|}
\hline & \multicolumn{2}{|c|}{$\mathrm{DC}$} & \multicolumn{2}{|c|}{ OS } & \multicolumn{2}{|c|}{$\mathrm{DC}$} & \multicolumn{2}{|c|}{ OS } \\
\hline & Adj. $R^{2}$ & $\overline{\mathrm{SE}}$ & Adj. $R^{2}$ & $\overline{S E}$ & Adj. $R^{2}$ & $\mathrm{SE}$ & Adj. $R^{2}$ & $\mathrm{SE}$ \\
\hline & \multicolumn{4}{|c|}{ (a) } & \multicolumn{4}{|c|}{ (c) } \\
\hline Trade numbers & 0.90 & 0.37 & 0.90 & 0.35 & 0.91 & 0.37 & 0.90 & 0.31 \\
\hline Trade volumes & 0.91 & 0.38 & 0.90 & 0.35 & 0.94 & 0.35 & 0.94 & 0.31 \\
\hline Opening positions & 0.90 & 0.35 & 0.91 & 0.35 & 0.92 & 0.35 & 0.93 & 0.31 \\
\hline \multirow[t]{2}{*}{ Closing positions } & 0.90 & 0.36 & 0.90 & 0.35 & 0.91 & 0.39 & 0.90 & 0.35 \\
\hline & \multicolumn{4}{|c|}{ (d) } & \multicolumn{4}{|c|}{ (e) } \\
\hline Trade numbers & 0.79 & 0.85 & 0.79 & 0.70 & 0.93 & 0.35 & 0.91 & 0.33 \\
\hline Trade volumes & 0.79 & 0.86 & 0.79 & 0.71 & 0.95 & 0.33 & 0.93 & 0.35 \\
\hline Opening positions & 0.78 & 0.83 & 0.80 & 0.72 & 0.93 & 0.35 & 0.89 & 0.37 \\
\hline \multirow[t]{2}{*}{ Closing positions } & 0.78 & 0.83 & 0.80 & 0.72 & 0.93 & 0.35 & 0.89 & 0.36 \\
\hline & \multicolumn{4}{|c|}{ (f) } & \multicolumn{4}{|c|}{ (g) } \\
\hline Trade numbers & 0.93 & 0.35 & 0.77 & 0.41 & 0.91 & 0.37 & 0.90 & 0.31 \\
\hline Trade volumes & 0.97 & 0.30 & 0.92 & 0.35 & 0.94 & 0.35 & 0.94 & 0.31 \\
\hline Opening positions & 0.92 & 0.35 & 0.77 & 0.39 & 0.92 & 0.35 & 0.93 & 0.31 \\
\hline \multirow[t]{2}{*}{ Closing positions } & 0.93 & 0.35 & 0.77 & 0.39 & 0.91 & 0.39 & 0.90 & 0.35 \\
\hline & \multicolumn{4}{|c|}{ (h) } & \multicolumn{4}{|c|}{ (i) } \\
\hline Trade numbers & 0.72 & 0.61 & 0.74 & 0.56 & 0.80 & 0.75 & 0.80 & 0.72 \\
\hline Trade volumes & 0.72 & 0.66 & 0.72 & 0.56 & 0.81 & 0.76 & 0.79 & 0.72 \\
\hline Opening positions & 0.73 & 0.57 & 0.74 & 0.57 & 0.80 & 0.73 & 0.80 & 0.74 \\
\hline \multirow[t]{2}{*}{ Closing positions } & 0.78 & 0.66 & 0.74 & 0.54 & 0.78 & 0.83 & 0.80 & 0.72 \\
\hline & \multicolumn{4}{|c|}{ (j) } & \multicolumn{4}{|c|}{ (k) } \\
\hline Trade numbers & 0.91 & 0.37 & -0.19 & 1.72 & 0.84 & 0.70 & -0.22 & 1.52 \\
\hline Trade volumes & 0.94 & 0.35 & 0.96 & 0.35 & -0.19 & 1.77 & -0.18 & 1.72 \\
\hline Opening positions & 0.91 & 0.37 & -0.19 & 1.72 & 0.84 & 0.70 & -0.22 & 1.52 \\
\hline Closing positions & 0.91 & 0.37 & -0.19 & 1.72 & 0.84 & 0.70 & -0.22 & 1.52 \\
\hline
\end{tabular}

Table 4: The adjusted $\mathrm{R}^{2}$ values of the fits and standard errors (SE), for the scaling laws measured under DC and OS events from (a) the dataset of EUR/USD transactions, the ABFXM populated with (c) ZI-DCT0, (d) GPAs, (e) $50 \%$ ZI-CV and 50\% ZI-DCT0 , (f) 70\% ZI-CV and 30\% ZI-DCT0, (g) $30 \%$ ZI-CV and 70\% ZI-DCT0, (h) ZI-DCT0 and GPAs ( $<50 \%$ ), (i) ZI-DCT0 and GPAs ( $\geqslant 50 \%$ ), (j) ZI-CV and GPAs $(<50 \%)$, (K) ZI-CV and GPAs $(\geqslant 50 \%)$. 


\begin{tabular}{|c|c|c|c|c|c|c|}
\hline & (b) & (c) & (d) & \multicolumn{3}{|c|}{ (e) } \\
\hline & ZI-CV & ZI-DCT0 & GPAs & ZI-CV & \multicolumn{2}{|c|}{ ZI-DCT0 } \\
\hline Min & $-100 \%$ & $-100 \%$ & $-99.50 \%$ & $-100 \%$ & \multicolumn{2}{|c|}{$-100 \%$} \\
\hline Max & $-99.90 \%$ & $3693.68 \%$ & $1169.86 \%$ & $-99.90 \%$ & \multicolumn{2}{|c|}{$921.84 \%$} \\
\hline Average & $-99.99 \%$ & $13.13 \%$ & $-27.49 \%$ & $-99.99 \%$ & \multicolumn{2}{|c|}{$921.84 \%$} \\
\hline Winning & 0 & $44.90 \%$ & $23.10 \%$ & 0 & \multicolumn{2}{|c|}{$47.08 \%$} \\
\hline Losing & $100 \%$ & $55.10 \%$ & $76.90 \%$ & $100 \%$ & \multicolumn{2}{|c|}{$52.92 \%$} \\
\hline & \multicolumn{2}{|c|}{ (f) } & \multicolumn{2}{|c|}{ (g) } & \multicolumn{2}{|c|}{ (h) } \\
\hline & ZI-CV & ZI-DCT0 & ZI-CV & ZI-DCT0 & GPAs & ZI-DCT0 \\
\hline Min & $-100 \%$ & $-100 \%$ & $-100 \%$ & $-100 \%$ & $-99.25 \%$ & $-99.97 \%$ \\
\hline Max & $-99.90 \%$ & $22.47 \%$ & $-99.90 \%$ & $711.02 \%$ & $38.42 \%$ & $419.75 \%$ \\
\hline Average & $-99.99 \%$ & $22.47 \%$ & $-99.99 \%$ & $-6.77 \%$ & $-29.63 \%$ & $-4.21 \%$ \\
\hline Winning & 0 & $45.40 \%$ & 0 & $42.69 \%$ & $31.58 \%$ & $34.08 \%$ \\
\hline \multirow[t]{3}{*}{ Losing } & $100 \%$ & $54.60 \%$ & $100 \%$ & $57.31 \%$ & $68.42 \%$ & $65.92 \%$ \\
\hline & \multicolumn{2}{|c|}{ (i) } & \multicolumn{2}{|c|}{ (j) } & \multicolumn{2}{|c|}{ (k) } \\
\hline & GPAs & ZI-DCT0 & ZI-CV & GPAs & ZI-CV & GPAs \\
\hline Min & $-99.79 \%$ & $-100 \%$ & $-100 \%$ & $-99.08 \%$ & $-100 \%$ & $-99.47 \%$ \\
\hline Max & $1169.86 \%$ & $780.16 \%$ & $-100 \%$ & $330.27 \%$ & $-100 \%$ & $258.66 \%$ \\
\hline Average & $-27.49 \%$ & $3.30 \%$ & $-100 \%$ & $-8.41 \%$ & $-100 \%$ & $-36.92 \%$ \\
\hline Winning & $23.10 \%$ & $40.73 \%$ & 0 & $30 \%$ & 0 & $14.25 \%$ \\
\hline Losing & $76.90 \%$ & $59.27 \%$ & $100 \%$ & $70 \%$ & $100 \%$ & $85.75 \%$ \\
\hline
\end{tabular}

Table 5: Statistics of the agents' ROI from the ABFXM populated with (b) ZI-CV, (c) ZI-DCT0, (d) GPAs, (e) 50\% ZI-CV and 50\% ZI-DCT0, (f) 70\% ZI-CV and 30\% ZI-DCT0, (g) 30\% ZI-CV and 70\% ZI-DCT0, (h) ZI-DCT0 and GPAs ( $<50 \%)$, (i) ZI-DCT0 and GPAs ( $\geqslant 50 \%)$, (j) ZI-CV and GPAs $(<50 \%)$, (k) ZI-CV and GPAs $(\geqslant 50 \%)$.

agents is extremely poor as all the agents achieved losses. The results indicate that the ZI-CV trading strategy may not be suitable for high-frequency trades. The reason behind the success of ZI agents in previous works such as Gode and Sunder (1993b); Sunder (2004); Tseng et al. (2010) may be due to the fact that these ZI agents trade in low frequency markets. When the ABFXM is populated with just ZI-DCT0 agents, case (c), the results are satisfactory and the average ZI-DCT0 agents' ROI is $13.13 \%$ while $44.90 \%$ of the agents achieve profits. Interestingly, and across all the simulation runs with different percentages of ZI-DTC0 agents present, such agents on average achieve better results (in terms of average and winning agents) than ZI-CV and GPAs. When the ABFXM is populated with just GPAs, case (d), the average ROI is $-27.49 \%$ while $23.10 \%$ of the agents achieve profits. The plain learning mechanism employed by the ZI-DCT0 is clearly more effective than the GP strategy which uses a series of technical indicators and fundamental analysis. The results offer evidence that a complex learning strategy may not be necessary for identifying investment and arbitrage opportunities in the financial market.

The results reported in Table 5 may be indicative, but require further exploration and analysis. The profitability of individual traders is not necessarily easy to establish. According to Olsen (2010), over $80 \%$ of traders in any one year achieve losses in their investments, and there is a possibility that the remaining $20 \%$ of traders lose money in their investment in the following year. This can be explained due to the difficulty of earning money in FX markets. Therefore, the results isolate that being able to identify DC and OS events in the price time series possibly the means to providing efficient decision support in the financial markets. The ROI results indicate that prices in financial markets exhibit "overshoots" and as a result a ZI-DCT0 agent mainly makes a profit when there are price overshoots. Thus, the results in Table 5 highlight the significance of considering events in studying the price time series based on intrinsic time rather than physical time changes. The computational costs which consist of the cost for evaluating the price data have to not be overlooked. For that reason, it has to be considered when it comes to choosing on an approach for identifying investment and arbitrage opportunities in financial markets.

In future work, we aim to establish a set of stylized facts regarding the real traders' investment profitability and performance. Such research will allow us to evaluate precisely the performance of different trading 
strategies.

\section{Discussion and Conclusions}

In this paper, we have studied the impact of the agents' trading strategies on the emergence of the stylized facts of trading activity in the high-frequency FX market. Understanding the market workings through stylized facts is fundamental for developing more effective decision support systems as well as strategies for autonomous trading agents. Three different type of agents have been studied: a variation of the ZI strategy with a constraint (ZI-CV) agents, ZI directional-change event (ZI-DCT0) agents, and genetic programming-based agents (GPAs).

To the best of our knowledge, the work presented in this paper, is the first study which has attempted to identify and understand the design effect of the agents' trading strategy in the emergence of the FX market stylized facts. Our work is different from the studies done in Cliff and Bruten (1997a); Tseng et al. (2010); Rayner et al. (2011); Gode and Sunder (1993a) in four major aspects. Firstly, we examine three alternative approaches to the design of the agents' strategies, two of which are commonly used for modelling agents in ABMs. Secondly, we examine the impact of the design of the agent's trading strategy on the emergence of the trading activity stylized facts in the high-frequency FX market, rather that examining the impact on the market mechanism as done in Cliff and Bruten (1997a); Tseng et al. (2010); Gode and Sunder (1993a) or on the stylized facts of the order flow as done in Rayner et al. (2011). Thirdly, our study focuses on the FX market, while the studies in Cliff and Bruten (1997a); Rayner et al. (2011); Gode and Sunder (1993a) use a double auction market and the study in Tseng et al. (2010) uses a web-based prediction market platform. Finally, to our knowledge, this is the first study that uses a unique high-frequency dataset of individual traders' historical transactions to validate the results of the agent-based models of traders.

The experimental results in section 5.3 show that the full set of stylized facts, as observed in the real FX market data, are reproduced by case (c) where all agents use the ZI-DTC0 strategy. The market setting (b) with the ABFXM being populated just with ZI-CV agents fails to generate the full set of stylized facts. The market setting (d) with just GPAs is able to reproduce the seasonality pattern in intraday data, but does not demonstrate the scaling laws, while the correlation behaviour of the trading activity generated is also not close to that exhibited in the real data. The other cases exhibited very limited aspects of the stylized facts, but not the full set. Examining the return on investment, the agents with the ZI-DTC0 strategy are clearly the most successful ones (in terms of the average as well as the number of winning agents).

Although the ZI-CV agents have advantages when it comes to investigating the effects of the market mechanism Daniel (2006); Gode and Sunder (1993a), from our experiments it appears that the randomness in the agents' trading activities and their inability to keep track of the overall market is the reason why the stylized facts are not exhibited. On the other hand, employing a complex strategy which involves learning does not seem to be able to reproduce the full set of stylized facts as observed in the real FX market data. This seems to provide further support to studies such as Tseng et al. (2010); Rayner et al. (2011); Daniel (2006); Gode and Sunder (1993a,b) that have argued that complex strategies, and in particular strategies that involve learning, may not be necessary nor sufficient for the emergence of phenomena such as stylized facts in markets. In our work, we have attempted to calibrate the parameters of the GPAs, but the wide variance in the decision rules that GPAs use does not allow us to pinpoint a single element of the GPAs' trading strategy mechanism as being one of the factors accountable for the non-emergence of the stylized facts. The complexity in the GPAs' trading strategy makes it difficult to draw out general conclusions regarding the results or inputs of the GPAs' trading activity in the market.

The ZI-DCT0 strategy avoids the complexity of GPAs and also the randomness of the ZI-CV strategy. The ZI-DCT0 agents are based on the directional change event approach and they respond to periodic patterns of a fixed size in the price time series. This relatively simple strategy appears to be effective in the HF FX market and the experiments suggest that this kind of behaviour is able to reproduce and explain the stylized facts observed in the real market and in comparison with the other strategies has also achieved better results in terms of ROI. The scaling law relations in the HF FX data also seem to suggest that these highly depend on the agents' responses to different price fluctuations. The results of this study suggest that a directional change event approach can aid our understanding of the workings and behaviour of the FX market. Our study also suggests that being able to recognise patterns in the time series such as DC and OS events may be key to providing effective decision support in the FX markets. The simple learning mechanism employed by the ZI-DCT0 is 
clearly more effective than the GP strategy which uses a series of technical indicators and fundamental analysis. Although, we cannot draw more general conclusions about the use of other learning strategies in the FX market, our results provide evidence that a complex learning strategy may not be necessary. The authors in LeBaron (2006); Hommes (2006); Samanidou et al. (2007); Daniel (2006); Duffy and Unver (2006); Gode and Sunder (1993a); Rayner et al. (2011) stress the importance of simplicity in building agent-based models in order to make possible the understanding of market behaviour and the forces driving the market. We believe that the ZI-DCT0 strategy allows us to gain a good insight into market behaviour dynamics without having to make various assumptions about the strategic behaviour of the agents.

Our findings could be used for identifying investment and arbitrage opportunities in the FX market either as part of a trading agent's strategy or as part of decision support tools. Our results provide evidence that introducing a threshold for the agents to trade when the price fluctuations exceed the threshold represents a key element in an agent's trading strategy in the HF FX. Hence, forecasting models can be constructed for predicting likely forthcoming price peaks or troughs as a means of aiding investment decisions. These forecasting models could make use of the stylized facts of the market (scaling laws) as a framework of signs to allow us to measure and characterise the level and flow of transactions and orders in the market. Such measurements would feed back into the investment strategies to enable them to adapt to changing market behaviour. Furthermore, a systematic risk management methodology can be developed based on the dynamic framework of the statistical properties derived from the scaling laws of financial market data.

Concerning the generalizability of the results, the simulations have been calibrated using stylized facts of the FX market transactions data, which can be grouped under three headings: seasonality, correlation statistics and scaling laws properties. These stylized facts were established using a transactions dataset provided by OANDA spanning over 2.25 years Aloud et al. (2012). The results reported have been checked for different sub-sets of data over the entire period. In addition, other studies reporting stylized facts have been used such as Dacorogna et al. (2001); Ito and Hashimoto (2006) to cross-reference these results.

There are several avenues for future work. Other strategies such as the Zero-Intelligence Plus (ZIP) Cliff and Bruten (1997b) and Adaptive Aggressiveness (AA) Vytelingum (2006) can be explored. The set up for our study involved the use of a real dataset which was fed into the simulation to facilitate the study of the emergence of stylized facts. An interesting line of work would be to remove the data feed and allow the market to evolve its own time price series and investigate a) its properties and b) the suitability and success rate of various strategies.

\section{Acknowledgments}

This research was funded by the Research Center of Humanities, Deanship of Scientific Research, King Saud University. We would like to thank the OANDA Corporation for providing the FX market datasets. We are very grateful to Prof E. Tsang, Prof R. Olsen and Dr A. Dupuis for their insightful suggestions and advice during the course of this study. We would also like to thank the anonymous reviewers for their useful comments and suggestions.

\section{References}

Alfi, V., M. Cristelli, L. Pietronero, and A. Zaccaria (2009), Minimal agent based model for financial markets I: origin and self-organization of stylized fatcs, The European Physical Journal B, 67(3), 385-397.

Aloud, M., E. Tsang, R. Olsen, and A. Dupuis (2011), A directional-change events approach for studying financial time series, Economics Papers, No 2011-28.

Aloud, M., M. Fasli, E. Tsang, A. Dupuis, and R. Olsen (2012), Modelling the high-frequency FX market: an agent-based approach, Tech. Rep. CES-519, University of Essex, United Kingdom.

Alvim, L., and R. Milidi $\tilde{A}^{o}$ (2013), Trading team composition for the intraday multistock market, Decision Support Systems, 54, 838-845.

An, B., V. R. Lesser, and K. M. Sim (2011), Strategic agents for multi-resource negotiation, Autonomous Agents and Multi-Agent Systems, 23(1), 114-153. 
Arthur, W. (1991), Designing economic agents that act like human agents: a behavioral approach to bounded rationality, American Economic Review, 81, 353-359.

Arthur, W. B., J. H. Holland, B. LeBaron, R. Palmer, and P. Tayler (1997), Asset pricing under endogenous expectations in an artificial stock market, in The economy as an evolving, complex system II, edited by W. Arthur, D. Lane, and S. Durlauf, pp. 15-44, Addison Wesley, Redwood City, CA.

Becker, G. (1962), Irrational behaviour and economic theory, Journal of Political Economy, 70, 1-13.

Chen, S.-H., and C.-H. Yeh (2001), Evolving traders and the business school with genetic programming: a new architecture of the agent-based artificial stock market, Journal of Economic Dynamics and Control, Elsevier, 25(3-4), 363-393.

Cliff, D., and J. Bruten (1997a), More than zero intelligence needed for continuous double-auction trading, Tech. Rep. HPL-97-157, HP Laboratories Bristol.

Cliff, D., and J. Bruten (1997b), Zero is not enough: On the lower limit of agent intelligence for continuous double auction markets, Tech. Rep. HPL-97-141, HP Technical Report.

Dacorogna, M., R. Genï¿œay, U. Mï̋œller, R. Olsen, and O. Pictet (2001), An introduction to high-frequency finance, Academic Press, San Diego.

Daniel, G. (2006), Asynchronous simulations of a limit order book, Ph.D. thesis, University of Manchester.

Duffy, J., and M. Unver (2006), Asset price bubbles and crashes with near-zero- intelligence traders, Economic Theory, 27, 537-563.

Farmer, J. (1998), Market force, ecology, and evolution, Industrial and Corporate Change, 11, 895-953.

Fasli, M., and Y. Kovalchuk (2011), Learning approaches for developing successful seller strategies in dynamic supply chain management, Inf. Sci., 181(16), 3411-3426.

Glattfelder, J., A. Dupuis, and R. Olsen (2011), Patterns in high-frequency FX data: discovery of 12 empirical scaling laws, Quantitative Finance, 11(4), 599-614.

Gode, D., and S. Sunder (1993a), Allocative efficiency of markets with zero intelligence (Z1) traders: market as a partial substitute for individual rationality, Journal of Political Economy, 101(1), 119-137.

Gode, D., and S. Sunder (1993b), Lower bounds for efficiency of surplus extraction in double auctions, in The Double Auction Market: Institutions, Theories, and Evidence. Santa Fe Institute Studies in the Sciences of Complexity, edited by D. Friedman and J. Rust, pp. 199-219, Perseus Publishing, Cambridge.

Hommes, C. (2006), Heterogeneous agent models in economics and finance, in Handbook of Computational Economics, Handbook of Computational Economics, vol. 2, edited by L. Tesfatsion and K. L. Judd, chap. 23, pp. 1109-1186, Elsevier.

Ito, T., and Y. Hashimoto (2006), Intraday seasonality in activities of the foreign exchange markets: Evidence from the electronic broking system, Journal of the Japanese and International Economies, Elsevier, 20, $637-664$.

Kettera, W., J. Collinsb, M. Ginib, A. Guptac, and P. Schraterb (2009), Detecting and forecasting economic regimes in multi-agent automated exchanges, Decision Support Systems, 47(4), 307-318.

Ladley, D. (2010), Zero-intelligence in economics and finance, Knowledge Engineering Review: Special issue on Agent Based Computational Economics, 27, 273-286.

LeBaron, B. (2001), A builder's guide to agent based financial markets, Quantitative Finance, 1 (2), 254-261.

LeBaron, B. (2006), Agent-based computational finance, in Handbook of Computational Economics, vol. 2, edited by L. Tesfatsion and K. L. Judd, 1 ed., chap. 24, pp. 1187-1233, Elsevier. 
Li, J., and E. Tsang (2000), Reducing failures in investment recommendations using genetic programming, Computing in Economics and Finance 2000 332, Society for Computational Economics, Barcelona, Spain.

LiCalzi, M., and P. Pellizzari (2003), Fundamentalists clashing over the book: a study of order-driven stock markets, Quantitative Finance, 3, 470-480.

Martinez-Jaramillo, S., and E. Tsang (2009), An heterogeneous, endogenous and co-evolutionary GP-based financial market, IEEE Transactions on Evolutionary Computation, 13(1), 33-55.

Niu, J., K. Cai, S. Parsons, P. McBurney, and E. Gerding (2010), What the 2007 tac market design game tells us about effective auction mechanisms, Journal of Autonomous Agents and Multiagent Systems, 21(2), 172-203.

Niu, J., K. Cai, S. Parsons, M. Fasli, and X. Yao (2012), A grey-box approach to automated mechanism design, Electronic Commerce Research and Applications, 11(1), 24-35.

Olsen, R. (2010), How to Trade, 1 ed., Think about Press, Olsen Ltd.

Pierre, B. (1965), Pareto (vilfredo) - cours d'ïœœconomie politique, Revue ïœconomique, Programme National Persï̈ce, 16 (5), 811-812.

Rayner, N., S. Phelps, and N. Constantinou (2011), Learning is neither sufficient nor necessary: a dynamic agent-based model of long memory in financial markets, in Workshop on Robustness and Reliability of Electronic Marketplaces.

Ren, F., M. Zhang, and K. (2009), Adaptive conceding strategies for automated trading agents in dynamic, open markets, Decision Support Systems, 46(3), 704-716.

Robinson, E., P. McBurney, and X. Yao (2012), Adaptive and Learning Agents, Lecture Notes in Computer Science, vol. 7113, chap. Co-learning segmentation in marketplaces, pp. 1-20, Springer Berlin Heidelberg, Berlin, Germany.

Samanidou, E., E. Zschischang, D. Stauffer, and T. Lux (2007), Agent-based models of financial markets, Reports on Progress in Physics, 70, 409-450.

Sanglier, M., M. Romain, and F. Flament (1994), A behavioral approach of the dynamics of financial markets, Decision Support Systems, 12(4-5), 405-413.

Serguieva, A., F. Liu, and P. Date (2011), Financial contagion simulation through modelling behavioural characteristics of market participants and capturing cross-market linkages, in IEEE Symposium on Computational Intelligence for Financial Engineering \& Economics, Paris, France.

Sim, K. (2002), A market-driven model for designing negotiation agents, Computational Intelligence, Special issue in Agent Technology for E-commerce, 18(4), 618-637.

Simon, H. (1982), Models of Bounded Rationality, MIT Press, Cambridge.

Simon, H. (1995), A behavioral model of rational choice, The Quarterly Journal of Economics, 69, 99-118.

Sunder, S. (2004), Market as an artifact aggregate efficiency from zero intelligence traders, in Models of a Man: Essays in memory of Herbert A. Simon, edited by M. Augier and J. March, pp. 501-519, MIT Press, Cambridge.

Tsang, E. (2010), Directional changes, definitions, Tech. Rep. 050-10, Centre for Computational Finance and Economic Agents (CCFEA), University of Essex, UK.

Tseng, J.-J., C.-H. Lin, C.-T. Lin, S.-C. Wang, and S.-P. Li (2010), Statistical properties of agent-based models in markets with continuous double auction mechanism, Physica A, 389, 1699-1707.

Vytelingum, P. (2006), The structure and behaviour of the continuous double auction, Ph.D. thesis, University of Southampton. 